\title{
A NOVEL MULTI-SCALE DOMAIN OVERLAPPING CFD/STH COUPLING METHODOLOGY FOR MULTI-DIMENSIONAL FLOWS RELEVANT TO NUCLEAR APPLICATIONS
}

\author{
T. P. Grunloh ${ }^{1}$, and A. Manera ${ }^{1}$ \\ ${ }^{1}$ Department of Nuclear Engineering and Radiological Sciences, University of Michigan-Ann Arbor, \\ 2355 Bonisteel Blvd., Ann Arbor, MI 48109, USA \\ grunloh@umich.edu,manera@umich.edu
}

\begin{abstract}
A novel multi-scale domain overlapping coupling methodology designed to couple a computational fluid dynamics (CFD) code with a system thermal hydraulic (STH) code was developed and its performance was investigated. The methodology has been implemented in the coupling infrastructure code Janus, developed at the University of Michigan, providing methods for the on-the-fly data transfer through memory between the commercial CFD code STAR-CCM+ and the US NRC best-estimate thermal hydraulic system code TRACE. Coupling between these two software packages is motivated by the desire to extend the range of applicability of TRACE to scenarios in which local momentum and energy transfer are important, such as three-dimensional mixing of localized slugs of deborated or cold water in the downcomer and lower plenum of a reactor pressure vessel. The intra-fluid shear forces necessary to correctly capture these effects are neglected in the TRACE equations of motion, but are readily calculated from CFD solutions. CFD/STH coupling implementations therefore have applications in reactor transients such as boron dilution scenarios, Anticipated Transient Without Scram (ATWS) and Main Steam Line Break (MSLB).

The proposed method is based on aliasing all spatial sources and sinks of momentum in the CFD domain as frictional losses in the system code domain. The internal velocity fields and, consequently, the inertial component of the pressure field are maintained consistent between the CFD and STH domains through a complementary velocity-matching interface. In this paper, coupled simulations are performed on Cartesian and cylindrical geometry with emphasis on consistency, convergence, and stability during transient scenarios. Results show that the presented domain overlapping coupling method is capable of adjusting pressure and velocity profiles of multi-dimensional system code solutions to match CFD solutions accurately. Important characteristics of transient simulations were found to include the background flow rate, specifically the stabilizing effect of viscous forces, as well as the time derivative of the flow rate. Under certain adverse conditions, the basic coupling method is found to produce unstable behavior. A stabilization method for adjusting CFD data is laid out and found to significantly improve the method's performance under the most challenging conditions. Recommendations are laid out for further improving the coupling via advanced time stepping methods.
\end{abstract}

KEYWORDS Multiscale, coupling, domain overlapping, CFD, STAR-CCM+ 


\section{INTRODUCTION}

\subsection{Motivation}

Although currently employed computational techniques have shown a remarkable ability to simulate the complex behavior of a Nuclear Power Plant (NPP) under a wide range of operational and accident conditions, the current trend of economically optimizing nuclear power plants (new designs, power uprates of operating reactors, etc.) motivates increased fidelity calculations for more accurate estimates of safety margins. The ability of system thermal hydraulic (STH) codes (i.e. TRACE (U. S. Nuclear Regulatory Commission 2010), RELAP5 (INL 2005), CATHARE (Tenchine, et al. 2012), and ATHLET (Lerchl, et al. 2012)) in the simulation of large networks of interconnected NPP components has been well established and documented in an ample body of literature. However, when three-dimensional effects play an important role in the NPP system, the constitutive relations and simplifying assumptions upon which STH codes rely break down. Specifically, best-estimate system codes are unable to correctly capture circulating flow patterns in large open regions, such as inlet plena without ad hoc modeling practices. Localized flow patterns can have a material impact in cases like anticipated transient without SCRAM, boron dilution, main steam line break, and pressurized thermal shock transients.

Recent years have seen Computational Fluid Dynamics (CFD) methods increasingly applied to single phase flow simulation in complex geometries relevant to nuclear engineering. To name just a few, Höhne et al. (Höhne, et al. 2008) simulated the mixing of de-borated slugs of water through the ROCOM facility, Lee et al. (Lee, et al. 2014) studied the effect of PWR internals on core inlet flow, and Jeong et al. (Jeong and Han 2008) simulated the flow field in downcomer and lower plenum of a PWR, explicitly accounting for reactor internals. The enduring trend of increasing availability of computational resources continues to add larger, higher resolution models to the class of tractable problems. Furthermore, researchers such as Merzari and Ninokata (Merzari and Ninokata 2011) and Prill and Class (Prill and Class 2014) have explored the use of Proper Orthogonal Decomposition (POD) to develop Reduced Order Models (ROM) for NPPs, potentially reducing the runtime burden significantly. Therefore, the coupling of CFD (or CFDlike ROMs) codes with best-estimate thermal-hydraulic system codes is a worthwhile endeavor, particularly in view of current industry inclinations towards power uprates, with the consequent reduction of safety margins, mandating a compensating increase in the fidelity of predictive models to satisfy safety regulations. In order for to maximize the value of STH-CFD coupling procedures, several conditions should be met. The correlations embedded in STH codes are often quite effective for the conditions under which they were created. Additionally, the employed CFD model must be fully validated or the coupled result cannot be trusted.

Primarily, two spatial coupling approaches are described in the literature, referred to as domain decomposition and domain overlapping. In the former, the system to be modeled is divided into discrete domains: one group simulated using the system code and the remaining domains simulated within the CFD code, connected through data exchange at the domain interfaces. In the domain overlapping approach, the entire system is computed within the system code, with selected regions further simulated within the CFD code as well. In this case, the CFD solution is used to correct the solution of the system code in the overlapped domain. The first coupling efforts for nuclear applications reported in the literature were from Aumiller et al. (Aumiller, Tomlinson and Bauer 2001) and Gibeling and Mahaffy (Gibeling and Mahaffy 2002), both works based on domain decomposition approaches. In recent years, several additional efforts have been focused on similar approaches. Anderson et al. (Anderson, Hassan and Schultz 2008) analyzed the Very High Temperature Reactor (VHTR). Bertolotto et al. (Bertolotto, et al. 2009) performed single phase mixing studies. Papukchiev et al. (A. Papukchiev, G. Lerchl, et al. n.d.) coupled ATHLET and ANSYS CFX and applied it to a Pressurized Thermal Shock Transient (A. Papukchiev, et al. 2011). Watanabe et al. (Watanabe, Anoda and Takano 2014) studied steam generator instability during station blackout conditions. $\mathrm{Li}$ et al. (Li, et al. 2014) coupled FLUENT and RELAP5 to study the Edwards-O'Brien blowdown problem as well as multi-loop flow through a 3D region. In some 
cases (Aumiller, Tomlinson and Bauer 2001) (Bertolotto, et al. 2009) (A. Papukchiev, G. Lerchl, et al. n.d.) (A. Papukchiev, et al. 2011), semi-implicit numerical coupling schemes are also described, aimed at relaxing the requirements on the integration time steps posed by explicit time-stepping methods, at the expense of increased computational costs. Additionally, implicit or semi-implicit methods generally require more data storage and stronger manipulation of each code's execution due to the need for timestep backups.

Researchers have also employed domain overlapping methods targeted at various phenomena. Fanning and Thomas (Fanning and Thomas 2010) used a CFD solution to correct the energy equation and the gravitational pressure term computed by the system code SAS4A/SASSYS-1 (Argonne National Laboratory 2012) for the simulation of sodium-cooled reactors. Jeltsov et al. (Jeltsov, et al. 2013) used a CFD solution to correct the system code energy equation through the implementation of a "virtual heater". Baviere et al. (Bavière, et al. 2014) (Pialla, et al. 2015) used a CFD solution to correct both momentum and enthalpy equations using iterative controls. In this case, a source/sink term in the momentum equation of the system code CATHARE is iteratively adjusted within a given time step until the overall pressure drop across the overlapped component is identical to the CFD solution.

Another coupling approach is to modify the closure coefficients used by the system code, taking advantage of pre-existing functionality. Cadinu and Kudinov (Cadinu and Kudinov 2009) employed such a method, but did not explicitly account for inertial effects on the pressure gradient, limiting the applicability of the method to steady state. The question therefore arises of how to use the CFD solution to correct the solution of the system code on-the-fly when inertial pressure effects are not negligible, without the need of additional iterations within the system code. We address this by deconstructing the pressure gradient to account for inertial effects and calculate a transient-compatible closure relation for the system code from on-demand CFD data. The coupling presented in this paper focuses on correcting the TRACE momentum equation.

In a previous paper (Grunloh and Manera, A novel domain overlapping strategy for the multiscale coupling of CFD with 1D system codes with applications to transient flows 2016), we established that domain overlapping methods can exhibit superior convergence characteristics to domain decomposition methods, at least in the explicit coupling regime. Specifically, this previous work showed that keeping the system code's mathematical system intact and consistent with the CFD system had a constructive effect on stability. The one-dimensional flow geometries presented in the previous paper were too simple to make any claims about performance with nuclear reactor geometries motivating us to extend the coupling treatment to the multi-dimensional formulation presented here. In this paper, we present two coupling test cases: a Cartesian case with flow primarily in the $x, y$ directions and a cylindrical test case with flow primarily in the $r, \theta$ directions. The cases are two-dimensional in the sense of the STH code, but the CFD models involved are fully three-dimensional. The geometries were chosen to be simple enough to allow useful theoretical analysis but complex enough to verify the coupling method under challenging conditions. In the cylindrical test case, transients involving large velocity time derivatives in otherwise low flow conditions proved exceptionally challenging, motivating the development of stability enhancing correction factors applied to data exchange between CFD to STH.

Most systems of engineering interest feature closed flow circuits. However, the cases presented herein are open flow circuits to allow for isolation of effects and clear theoretical analysis. Application to closed loops with feedback effects is the subject of ongoing work. Buoyant flows are also of high importance in nuclear reactor safety. The authors have applied domain overlapping methods to simplified thermosiphon flows (Grunloh and Manera 2014). Although the methods presented in this paper were developed such flows in mind, their application to multi-dimensional buoyant flows is a subject of future inquiry.

The domain overlapping coupling methods developed in this paper are targeted towards transient conditions in nuclear reactors characterized by some form of 3-D mixing. One such transient is the main steam line break (MSLB) transient in which the steam line connected to one of the steam generators 
ruptures causing overcooling in the associated primary loop and leading therefore to an asymmetric coolant temperature distribution in the lower plenum of the reactor. Another such transient is the Anticipated Transient without SCRAM (ATWS) where the reactor shutdown is achieved by injecting a neutron poison (borated water).

The MSLB transient begins with the double-ended rupture of one steam line, resulting in depressurization of the secondary side of the affected steam generator, a consequent increase of the heat transfer from the primary side to the secondary side, and eventually to a decrease in temperature of the primary coolant in the affected loop. The colder primary water will translate into a reactivity insertion once it reaches the core. The extent of the reactivity insertion and the location where a power peak occurs will depend on how the colder coolant will mix with the coolant flowing from the other primary loops, before reaching the core inlet. In the late 1990s, an international benchmark study was organized to study the effects of the transient core behavior during MSLB (Ivanov, et al. 1999). MSLB transients have received a large amount of attention from the nuclear power industry as well (Grundmann and Kliem 2003) (Kozlowski, et al. 2004) (Joo, et al. 2003).

\subsection{Nomenclature}

Table 1. Nomenclature used in present paper.

\begin{tabular}{|l|l|}
\hline \multicolumn{2}{|c|}{ Variable } \\
\hline$\rho$ & Density \\
\hline$K$ & Wall friction coefficient \\
\hline$D h$ & Hydraulic diameter \\
\hline$f F$ & Fanning friction factor \\
\hline$V$ & Velocity \\
\hline$V$ & Intermediate velocity \\
\hline $\mathbf{v}$ & Vector velocity \\
\hline $\mathbf{n}$ & Outward facing normal vector \\
\hline $\mathbf{T}$ & Stress tensor \\
\hline$p$ & Pressure \\
\hline$p$ & Volume averaged pressure \\
\hline & TRACE cell length \\
\hline$\Delta x$ & Distance between adjacent cell centers \\
\hline$\Delta t$ & Time step \\
\hline$\Delta p$ & Change in pressure \\
\hline$d \Delta p$ & Change in pressure drop between two cells \\
\hline$\Delta \Delta P$ & Total change in pressure drop during timestep \\
\hline$E \Delta \Delta p$ & Error in $\Delta \Delta p$ from not using stabilizing term \\
\hline$m$ & Mass flow rate \\
\hline$t$ & Time \\
\hline$\beta$ & Upwind parameter \\
\hline
\end{tabular}




\begin{tabular}{|c|l|}
\hline$R$ & Average radius \\
\hline$A$ & Area \\
\hline \multicolumn{2}{|c|}{ Subscripts } \\
\hline in & Inlet \\
\hline out & Outlet \\
\hline$N I$ & Non-Inertial \\
\hline$j$ & Cell $j$ \\
\hline$j+1 / 2$ & Edge between cells $j$ and $j+1$ \\
\hline tot & Total \\
\hline$i \rightarrow j$ & From cell $i$ to cell $j$ \\
\hline$I$ & Inertial component \\
\hline$\alpha$ & References $\alpha$ direction \\
\hline$\Omega$ & Bounding surface \\
\hline \multicolumn{2}{|c|}{ Superscripts } \\
\hline$V$ & Volume averaged \\
\hline$S$ & Surface averaged \\
\hline$n$ & Time step index \\
\hline$m$ & Newton-Raphson iteration number \\
\hline$M$ & Maximum number of Newton iterations \\
\hline$\perp$ & Perpendicular component \\
\hline \multicolumn{2}{|c|}{ Acronyms } \\
\hline IDO & Inertial Domain Overlapping \\
\hline SIDO & Stabilized Inertial Domain Overlapping \\
\hline CV & Control Volume \\
\hline CFD & Computational Fluid Dynamics \\
\hline STH & System Thermal Hydraulic \\
\hline VMF & Velocity Matching Face \\
\hline CCM & STAR-CCM + \\
\hline TRC & TRACE \\
\hline \multicolumn{2}{|c|}{} \\
\hline
\end{tabular}

\section{THEORY}

The ideas underlying the approach taken to "correcting" STH momentum conservation equations using accompanying CFD solutions are discussed in this section under the assumption that a fully validated CFD model of the coupled region already exists. In $\$ 2.1$ and $\$ 2.2$, we broadly discuss how TRACE solves flow equations, singling out specific parameters to be targeted for coupling. In $\$ 2.3$ and $\S 2.4$, we outline the approach of using CFD solutions to adjust the STH solution. 


\subsection{STH Momentum Conservation}

Under isothermal incompressible three-dimensional single phase liquid water flow conditions (applicable to test cases used here), the discretized TRACE momentum equation in the $\alpha$ direction (where $\alpha$ can be $x, y, z, r$, or $\theta$ ) reduces to Eq. 1 (U. S. Nuclear Regulatory Commission 2010), along with analogous equations for the other two accompanying directions:

$$
\begin{gathered}
V \alpha, i+12, j, k n+1-V \alpha, i+12, j, k n \Delta t+V \cdot \nabla V \alpha, i+12, j, k=-1 \rho p i+1, j, k n+1-p i, j, k n+1 R \alpha \Delta \alpha i+12, j, k-K \alpha, \\
i+12, j, k n 2 V \alpha, i+12, j, k n+1-V \alpha, i+12, j, k n V \alpha, i+12, j, k n,
\end{gathered}
$$

where $V \alpha, i+12, j, k n$ is the $\alpha$-component of velocity at the edge between adjacent cells with indices $i, j, k$

and $i+1, j, k, p i, j, k$ is the volume-averaged pressure of cell $i, j, k, \rho$ is the mass density, $\Delta t$ is the timestep, $\Delta \alpha$ is the mesh size in the $\alpha$ direction, and $R \alpha$ is a length multiplier that is unity for all directions except $\alpha=\theta$ in which $R \alpha$ is the radius at which the cell centers lie. All three directions are coupled through the convective acceleration terms $V \cdot \nabla V$, as well as through mass conservation, or continuity This formulation is valid for both Cartesian and cylindrical coordinate systems. The effects of friction and turbulence are modeled through the coefficient $K \alpha, i+12, j, k n$ which is calculated through correlations such as the Churchill correlation (U. S. Nuclear Regulatory Commission 2010). For brevity, unnecessary indices will be omitted for the remainder of this paper. Comparison to the continuous Navier-Stokes equations makes clear the role of the coefficient as a model for the divergence of the stress tensor $\mathbf{T}$, including both viscous and turbulent stresses. As shown in Eq. 2 the $K$ coefficient can be related to the Fanning friction factor $f F$ used in TRACE (not to be confused with the Darcy friction factor which is 4 times larger):

$$
-\nabla \cdot \mathbf{T} \rho \rightarrow K V 2=2 f F R \alpha \Delta \alpha D h V 2
$$

The friction factor can be written as a normalized pressure gradient as shown in Eq. 3 (Bergman, et al. 2011).

$$
f F=-\nabla p \bullet e \alpha \text { fricDh } 2 \rho V 2
$$

where $D h$ is the hydraulic diameter, $\rho$ is the fluid density (water in this report), $\boldsymbol{e} \boldsymbol{\alpha}$ is a unit vector in the $\alpha$ direction, and $V$ is the mean fluid velocity. A primary objective of the proposed coupling method is to adjust the TRACE momentum equation in a specific region to reproduce the results of higher fidelity CFD models. Rather than accomplishing this through adding a momentum source to Eq. 1, we instead modify friction factors in TRACE for three reasons: 1) the friction factor is essentially a normalized pressure gradient and is thus easily interpreted, 2) the friction factors used in TRACE are evaluated at the previous time step, making it straightforward to modify the term in a consistent way, and 3) the stability of TRACE has already been thoroughly studied by its developers for the friction factor as currently implemented; utilizing the same structure allows us to leverage this work.

STH codes such as TRACE often use a staggered grid discretization, defining scalar quantities like pressure and temperature, interpreted as averages over the control volumes (CVs), in the center of the 
CVs (Idaho National Engineering and Environmental Laboratory 2001) (U. S. Nuclear Regulatory Commission 2010). Defined at the boundaries of the CV are vector quantities such as velocity, as well as friction factors that account for the frictional pressure difference between adjacent cells. With staggered grids, interpolation is necessary to calculate the velocity at the center of a CV or the pressure at the CV boundary.

\subsection{TRACE Solution by Nonlinear Iteration}

The general outline of the TRACE solution procedure is given in Fig. 1.

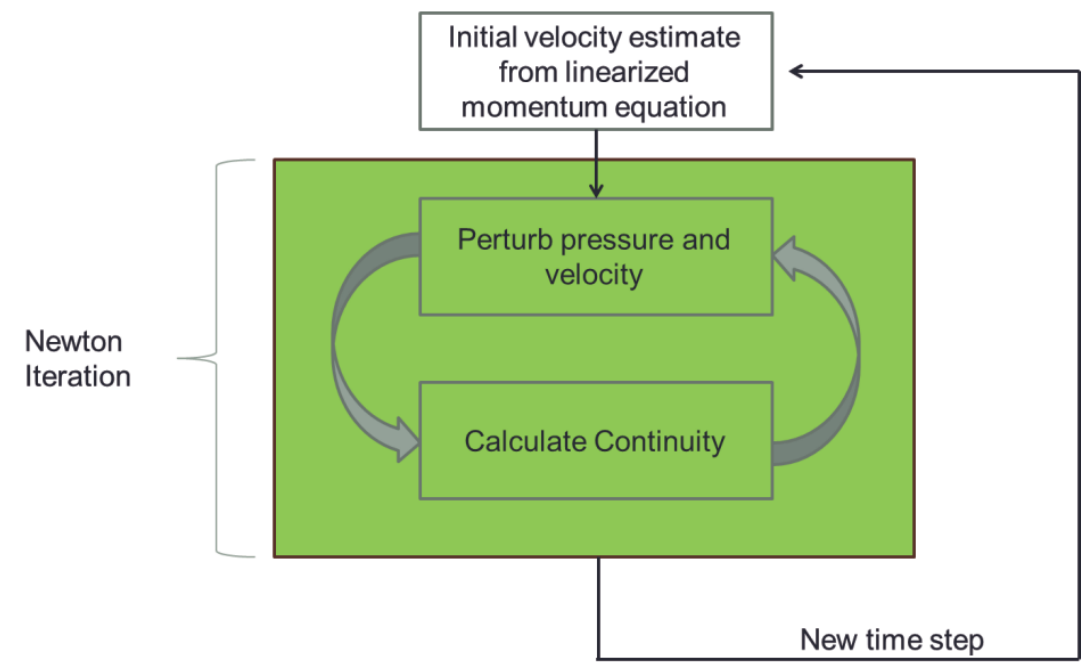

Fig. 1. Schematic of nonlinear iteration used in the TRACE solution procedure

TRACE begins a time step by calculating an initial estimate of the new time velocity $(\operatorname{V\alpha n}+1,0$, where the $O$ superscript signifies the initial, unadjusted estimate and the tilde represents the intermediate nature of the value) from a version of Eq. 1 built from previous time step pressures (i.e. replace $p n+1$ with $p n$ in Eq. 1). As shown in Eq. 4, this equation is linear in $V \alpha n+1,0$ and can be solved directly:

$$
\begin{gathered}
V \alpha n+1,01+2 \Delta t K \alpha, i+12, j, k n=V \alpha, i+12, j, k n-\Delta t 1 \rho p i+1, j, k n-p i, j, k n R \alpha \Delta \alpha i+12, j, k-K \alpha, i+12, j, k n V \alpha \\
, i+12, j, k n V \alpha, i+12, j, k n .
\end{gathered}
$$

After calculating $V \alpha n+1,0$, the variable given by Eq. 5 is stored, characterizing the relationship of changes in pressure drop between adjacent cells and the velocity across the edge shared by the adjacent cells:

$$
d V d \Delta p j+12=\Delta t \rho R \alpha \Delta \alpha 1+\Delta t 2 K j+12 n V j+12 n+\beta \nabla j+12 V j+12 n,
$$

where $\beta$ is an upwinding parameter dependent on flow direction. Eqs. 4 and 5 are built from a linearized version of the momentum equation. The fully nonlinear equations of motion are solved with a NewtonRaphson iteration cycle, which can be conceptualized as adjusting the pressure field based on a new 
velocity estimate, followed by adjusting the velocity field based on the newest pressure field estimate. Specifically, a set of equations is built by perturbing the pressure and velocity variables in the mass and energy conservation equations with the as yet unknown pressure perturbation $\delta p j m$. The pressure variable is replaced with Eq. 6:

$$
p j n+1, m+1=p j n+1, m+\delta p j m .
$$

where $j$ refers to a cell and $m$ refers to the iteration number of the Newton cycle. The velocity perturbation shown in Eq. 7 is built from the pressure perturbation using Eq. 5:

$$
V j+12 n+1, m+1=V j+12 n+1, m+d V d \Delta p j+12 \delta p j m-\delta p j+1 m \text {. }
$$

For incompressible isothermal flow, the linearized mass conservation equation with perturbed values is shown in Eq. 8:

$$
d V d \Delta p j+12 \delta p j m-\delta p j+1 m-d V d \Delta p j-12 \delta p j-1 m-\delta p j m \Delta x=V j+12 n+1, m-V j-12 n+1, m \Delta x .
$$

The 3-D version is built by adding analogous terms for the remaining two directions. Applying the perturbed balance equation to each cell yields a system of expressions to be solved simultaneously for the pressure perturbations $\delta p j m$, which are used to update the pressure and velocity fields. This cycle continues until convergence, given by $(\lim m \rightarrow \infty V \alpha n+1, m=V \alpha n+1, \lim m \rightarrow \infty \delta p j m \rightarrow 0)$, or a maximum number of iterations is reached.

\subsection{Coupled Friction Factor Formulation}

In this section, the principles of friction factor coupling developed in $\$ 2.3 .1$ are applied to the solver described in $\S 2.2$ in order to construct two domain overlapping approaches. A base formulation, referred to as the Inertial Domain Overlapping (IDO) coupling method is built by forming a non-inertial pressure gradient and directly applying that to the coupled friction factor calculation. A more advanced method, referred to as the Stabilized Inertial Domain Overlapping (SIDO) coupling method, is formulated by performing a deeper analysis of the mathematical structure of the TRACE solver and correcting for secondary effects of the coupling on the TRACE flow solver. The two methods are found to behave quite similarly, except for challenging conditions in which the SIDO method exhibits superior stability properties.

\subsubsection{Computing Friction Factors from CFD data}

Starting from the continuous Navier-Stokes equations, a pressure gradient vector-valued function can be defined as a combination of three terms as shown in Eq. 9. The first term on the right of the equality, the inertial contribution, describes the effects of unsteady velocities. The second term captures the effects of acceleration due to velocity gradients and is referred to as the acceleration or convective pressure gradient. Finally, the frictional pressure gradient resulting from viscous dissipation is the third term on the right. In cases of turbulent flow, consider $\mathbf{v}$ to be the Reynolds-averaged velocity and for $\mathbf{T}$ to include Reynolds stresses: 


$$
\nabla p=-\rho \partial \mathbf{v} \partial t-\rho \mathbf{v} \cdot \nabla \mathbf{v}+\nabla \cdot \mathbf{T}
$$

The TRACE momentum balance of Eq. 1 is comprised of directly analogous terms, each of which must be specifically addressed by a successful coupling formulation. In TRACE, the convective term, $V \cdot \nabla V x, i+12, j, k$ in Eq. 1 is easily eliminated from the momentum equation for edges in the coupled region. Similarly, the term $\beta \nabla j+12 V j+12 n$ can be easily omitted from Eq. 5. The combined effects of friction, turbulence, and convective acceleration computed with CFD are therefore combined into the friction coefficient used in TRACE. The inertial term, however, is a byproduct of the calculation procedure of Eqs. 1, 6, and 7 and cannot be excluded from TRACE. The term is instead corrected through velocity field manipulation via the velocity-matching functionality introduced in $\$ 2.3 .2$. Therefore, only the non-inertial (NI) pressure drop terms (acceleration and friction) are to be corrected through a modified friction coefficient. Based on this, a NI pressure gradient is defined by subtracting the inertial contribution from the total pressure gradient:

$$
\nabla p N I=\nabla p t o t+\rho \partial \mathbf{v} \partial t
$$

Recall that the friction factor can be interpreted as a normalized pressure gradient, and that TRACE pressure is consistent with a volume-averaged pressure in STAR-CCM+. A friction factor computed from CFD data must thus be based on changes in volume-averaged pressure with respect to the volumes that make up the TRACE nodalization. The geometric definitions of TRACE are automatically passed to STAR-CCM+, which uses the data to overlay the TRACE mesh on the STAR-CCM+ mesh. Specifically, the Janus interface that couples STAR-CCM+ with TRACE builds data structures with elements for each cell and edge of the TRACE geometry.

During coupling startup procedures, sub-surfaces are automatically built in the CFD model for each cell face of the 3D system. During the time between STAR-CCM+ and TRACE execution, surface integrals are performed over the faces to calculate the area-averaged CFD velocity analogous to the TRACE velocity across the same edge.

Once values for the velocity normal to each cell face and average pressure of each cell are calculated and stored, it is possible to calculate the non-inertial pressure gradients needed for the volumetric coupling. The first step is to calculate the volume-averaged pressure for each cell. Next $\Delta p i \rightarrow j$, the pressure drop across the edges defined by each pair of adjacent cells $V i$ and $V j$ is calculated as shown in Eq. 11:

$$
\Delta p i \rightarrow j=\operatorname{Vip} x d 3 x-\operatorname{Vjp} x d 3 x
$$

The portion of the pressure drop resulting from inertia, $\Delta p I, \alpha$, is then estimated from area-averaged velocities as shown in Eq. 12:

$$
\Delta p I, \alpha=-R \alpha \Delta \alpha V 2+\Delta \alpha V 12 \rho \Delta t 1 A \Omega \Omega v \alpha n+1 d \mathrm{~S}-\Omega v \alpha n d S
$$

where $\alpha$ again refers to any coordinate among $x, y, z, r, \theta, \Omega$ refers to the surface defining the edge, $A \Omega$ is the surface area of the edge, while $R \alpha=$ max + rmin 2 for the $\theta$ coordinate (where rmax is the radius defining the upper bound of the cell and rmin defines the lower bound) and $R \alpha=1$ for all other 
coordinates $x, y, z, r$. A non-inertial friction factor for use in the STH momentum equation for coupled components can then be formed as shown in Eq. 13:

$$
f N I, i \rightarrow j=\Delta p N I \Delta s \alpha D h 2 \rho V 2=\Delta p i \rightarrow j-\Delta p I, \alpha \Delta s \alpha D h 2 \rho V 2,
$$

where $\Delta s \alpha$ is shorthand for the distance between centers of the two cells adjoining the edge:

$$
\Delta s \alpha=R \alpha \Delta \alpha V 2+\Delta \alpha V 12
$$

In coupled simulations, $\Delta p N I$ is the actual value passed to TRACE from STAR-CCM+ and all other variables in Eq. 13 are as defined in TRACE.

\subsubsection{Velocity Field Coupling}

A general velocity field is determined based on three primary influences: boundary conditions, continuity, and the pressure field. Through these mechanisms, a pressure-velocity feedback is established, an example of which is higher velocities yielding higher frictional losses. In a coupled simulation, where TRACE friction factors are specified from an outside source, this feedback is disrupted. For example, in a coupled steady-state simulation, any nonzero velocity across a face can produce any pressure drop across that face, depending only on the friction factor. The consequences of this effect are further explored in $\S 3$.

"Velocity Matching Faces" (VMFs) are implemented in Janus to reinstate the pressure-velocity feedback. Ideally, a VMF applied to a specific edge causes TRACE to calculate the same velocity as the equivalent STAR-CCM+ edge. VMFs are user-specified for an array of faces such that consistency is enforced between TRACE and STAR-CCM+, but also such that enough faces are left unconstrained to allow TRACE to independently enforce mass conservation and avoid problem over-specification. For VMF configurations that fully dictate the flow field, the effects of the specific configuration are minimal, as will be demonstrated.

As a prelude to discussing the specific VMF implementation, the Newton iteration implemented in TRACE is more closely examined. Eq. 7 is rearranged to form Eq. 15, an expression for the change in pressure drop across an edge at the $m t h$ step of the iteration:

$$
d \Delta P j+12 m=\delta p j m-\delta p j+1 m=V \alpha, j+12 n+1, m+1-V \alpha, j+12 n+1, m d V d \Delta p j+12 .
$$

The relationship for the new time pressure drop across the edge is shown in Eq. 16, calculated by summing over all inner iterations:

$$
\begin{gathered}
\Delta P j+12 n+1 \\
=\Delta P j+12 n+m=1 M d \Delta P j+12 m=\Delta P j+12 n+d V d \Delta p j+12-1 V \alpha, j+12 n+1-V \alpha, j+12 n+1,0, \quad 16
\end{gathered}
$$

where $M$ is the maximum number of inner iterations allowed by TRACE. Restated, Eq. 16 represents two useful relationships. First, the difference between the initial estimate of the velocity and the final new time velocity is shown in Eq. 17: 


$$
V \alpha, j+12 n+1-V \alpha, j+12 n+1,0=\Delta P j+12 n+1-\Delta P j+12 n d V d \Delta p j+12 .
$$

Additionally, the total change in pressure drop in a time step $(\Delta \Delta p)$ is shown in Eq. 18:

$$
\Delta \Delta p j+12=d V d \Delta p j+12-1 V \alpha, j+12 n+1-V \alpha, j+12 n+1,0 .
$$

In the IDO coupling method, $V \alpha, j+12 n+1,0$ is replaced with a velocity calculated from STAR-CCM+ for the analogous edge, $V C F D$. The new time velocity can then be estimated from Eq. 17 as shown in Eq. 19:

$$
V \alpha, j+12 n+1=V C F D+\Delta P j+12 n+1-\Delta P j+12 n d V d \Delta p j+12
$$

Generally $d V d \Delta p j+12$ is very small and $V \alpha, j+12 n+1 \approx V C F D$. The IDO method does not further consider the consequences of $V \alpha, j+12 n+1,0$ replacement. The VMF implementation used in the SIDO method is realized by instead replacing $V \alpha, j+12 n+1,0$ with $V 1$, a "corrected" velocity defined in Eq. 20:

$$
V 1=V C F D-\Delta P j+12 n+1-\Delta P j+12 n \Delta t \rho \Delta s \alpha 1+2 \Delta t K j+12 n V j+12 n .
$$

Performing this substitution in Eq. 17 leads to Eq. 21, suggesting that the new time velocity can be driven to match the CFD velocity, assuming that the value used for $\Delta P j+12 n+1-\Delta P j+12 n$ is accurate:

$$
V \alpha, j+12 n+1-V C F D=0
$$

The SIDO method further considers the action of $V \alpha, j+12 n+1,0$ substitution on the pressure drop change in a time step, an expression for which can be calculated by subtracting the value of $\Delta \Delta p$ that results from a VMF from the unaffected equivalent, shown in Eq. 22:

$$
E \Delta \Delta p=V \alpha, j+12 n+1,0-V 1 d V d \Delta p j+12 \text {. }
$$

The $E \Delta \Delta p$ term can be used to calculate a stabilized friction factor for VMF faces as shown in Eq. 23: 


$$
f N I=\Delta p t o t-\Delta p I, \alpha-E \Delta \Delta p \Delta s \alpha D h 2 \rho V 2 \text {. }
$$

The stabilized friction factor used in the SIDO method adjusts the pressure drop incoming from STAR$\mathrm{CCM}+$, already augmented to account for inertial contributions, to mitigate the effects of VMF application on pressure drop across the affected edge.

In order to properly calculate $E \Delta \Delta$, Eq. 22 must be evaluated simultaneously with Eqs. 4 and 5, since the $K$ coefficient in the latter equations depends on $f N I$ which depends on $E \Delta \Delta p$. In TRACE, these three equations are linear in their unknowns $E \Delta \Delta p, V \alpha, j+12 n+1,0, d V d \Delta p j+12$, and algebraic manipulation can yield an explicit expression for $E \Delta \Delta p$ with the specific form dependent on a number of variables internal to TRACE.

\subsubsection{Continuity with Disparate Meshes}

To model the flow of water, TRACE and STAR-CCM+ both solve mass, momentum, and energy conservation equations based on the same first principles, but the starkly different degree of scales resolved by the codes must be considered. The version of continuity, or mass conservation, enforced by CFD codes for incompressible flow is best conceptualized as the differential version of Eq. 24, describing a solenoidal field in the flow domain:

$$
\nabla \cdot \mathrm{v}=0
$$

The integral continuity equation of Eq. 25, employed by TRACE, can be derived from the differential form by application of the divergence theorem:

$$
i \operatorname{Siv} \bullet \mathbf{n} d S=i v i \perp A i n i=0
$$

where the summation is over the number of faces bounding a particular cell, with $n i$ equal to \pm 1 depending on which side of the cell the $i t h$ face lies on, and $v i \perp$ signifies the velocity perpendicular, or flowing through, the $i t h$ face with area $A i$. While Eqs. 24 and 25 are equivalent for a continuous velocity field, there can be important differences when mapping data between meshes of different resolution. For instance, the flow areas in TRACE are defined in user input and are therefore exact. Meanwhile, the equivalent flow areas in STAR-CCM+ are calculated based on the finite volume mesh and subject to interpolation error. In general, STH flow areas and their CFD equivalents might differ by a small amount, depending on how fine the CFD mesh is.

Using an appropriate VMF configuration allows TRACE to correct continuity by adjusting the velocity of unconstrained faces. However, during certain types of challenging transients (discussed in §4.4.2), the TRACE corrections can become rather abrupt, which can in turn have a strong effect on the inertial pressure gradient. The effects of VMF configurations are analytically explored in $\$ 4.3 .1$ and the importance of explicitly accounting for STH continuity are shown numerically in Fig. 36.

\subsubsection{Domain Overlapping Method Summary}

The IDO and SIDO methods, distinguished by friction factor and VMF formulations, are summarized in Table 2.Both methods predict the inertial contribution to the pressure drop between two adjacent cells to remove it from the total pressure drop to calculate a non-inertial friction factor. Both methods also seek to 
allow for velocity field and inertial pressure consistency through VMFs. The SIDO method includes extra correction factors that more closely integrate it into the TRACE nonlinear iteration solution procedure.

Table 2. Summary of formulations for the Inertial Domain Overlapping (IDO) coupling method and the Stabilized Inertial Domain Overlapping (SIDO) coupling method.

\begin{tabular}{|c|c|c|}
\hline $\begin{array}{c}\text { Meth } \\
\text { od }\end{array}$ & Friction Factor & Velocity Matching Face \\
\hline IDO & $\begin{array}{c}f N I, i \rightarrow j=\Delta p i \rightarrow j-\Delta p I, \alpha \Delta s \alpha D h \\
2 \rho V 2\end{array}$ & $V \alpha, j+12 n+1,0 \rightarrow V C F D$ \\
\hline SIDO & $\begin{array}{c}f N I, i \rightarrow j=\Delta p i \rightarrow j-\Delta p I, \alpha-E \Delta \Delta p \\
\Delta s \alpha D h 2 \rho V 2\end{array}$ & $\begin{array}{c}V \alpha, j+12 n+1,0 \rightarrow V C F D-\Delta P j+12 n+1-\Delta P j+12 n \Delta t \rho \Delta s \alpha \\
1+2 \Delta t K j+12 n V j+12 n\end{array}$ \\
\hline
\end{tabular}

\subsection{Data Mapping}

It is beneficial to discuss specifically how the data are calculated, as well as the geometry of data transfer. In $\$ 2.4 .1$ the specifics of transferring multi-dimensional data to a $1 \mathrm{D}$ domain are discussed, while the inverse issue is discussed in $\$ 2.4 .2$.

\subsubsection{D to 1D Data Transfer}

An adjacent pair of representative cells is shown in Fig. 2 along with their shared edge for a cylindrical configuration. In this figure, the TRACE nodalization is shown on the right with the CFD equivalent on the left. As indicted in the figure, a volume-averaged pressure is calculated for a region of the CFD mesh corresponding to a TRACE node. Additionally, an area-integrated velocity (units of volume over time) is calculated for a planar section corresponding to the face between the cells. These three quantities are combined to calculate a non-inertial friction factor that is passed to TRACE.

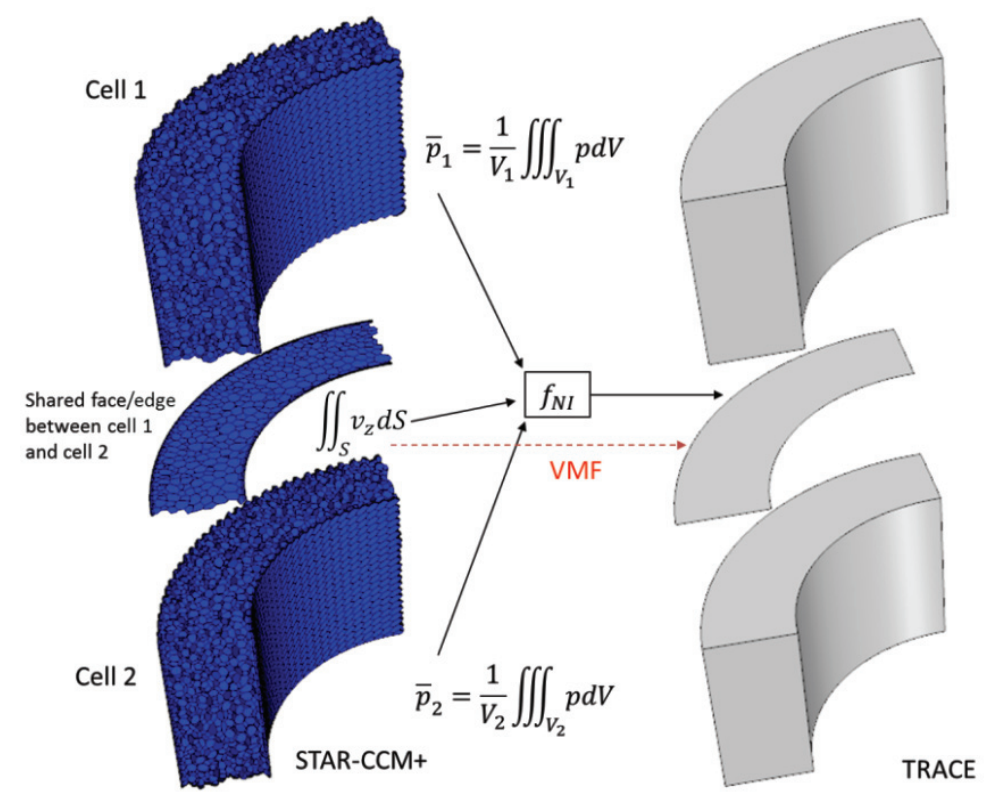

Fig. 2. Data transfer schematic for VESSEL coupling interface. 
The diagram in Fig. 2 further demonstrates that the IDO and SIDO coupling methods affect only edges, since the coupling method seeks to correct pressure differences rather than absolute pressures. The diagram also demonstrates the disparity in mesh resolution. Even with the relatively coarse CFD mesh pictured in Fig. 2, there are hundreds to thousands of CFD cells per each TRACE volume. Under such circumstances, the CFD solution can be essentially considered to be continuous in comparison to the STH solution, and only the TRACE discretization is discussed in detail in this paper.

\subsubsection{D to 3D Data Transfer}

The problem of transferring data from lower to higher dimensionality is a common theme in coupling discussion because additionally information is required. In this paper, the profiles at CFD boundaries are taken to be flat. Specifically, a mass flow rate passed from TRACE to STAR-CCM+ is distributed evenly across the CFD inlet. Flat turbulence quantities are also used. These assumptions introduce uncertainty into the coupled model. For the cases presented here, the CFD boundaries are taken to be sufficiently far from the region of interest to allow the flow to develop, minimizing these effects. For more advanced coupling cases, it may be necessary to pay special attention to the 1D-to-3D data transfer issues discussed here.

\section{Cartesian flow splitter}

The Cartesian geometry shown in Fig. 3 was designed as an initial verification case for the IDO coupling method and as a demonstration for VMF application. Referred to as a "flow splitter", the geometry includes two possible flow paths, designated as the lower and upper legs, as well as four changes in stream direction, two dictated by geometry and two by pressure balance. The TRACE geometry is only one cell deep in the $z$ direction, so no open mixing regions exist and the model is effectively twodimensional. Each cell is cubic with dimensions of $0.1 \mathrm{~m} \times 0.1 \mathrm{~m} \times 0.1 \mathrm{~m}$. While the STAR-CCM+ model is three-dimensional, there are no nonzero flow faces in the $z$ direction in the TRACE model and so the coupled case is not considered to be fully three-dimensional. The quality of the coupling is quantified with the pressure drop between the inlet and outlet ( $\Delta p=p i n-p o u t$, as indicated in Fig. 3) as well as the mass flow through each leg.

The dual flow path configuration of this geometry provides an ideal introduction to the VMF functionality and its verification. The flow through each leg is determined by the balance between the frictional and form losses incurred along each path. The coupling method used here does not allow TRACE to influence the friction factor and therefore disrupts this balance. However, guiding the velocity field with VMFs reinstates the pressure-velocity feedback through the CFD solution. Because only two possible flow paths exist, one VMF is sufficient to determine flow through both legs, as the conservation of mass will ensure the non-VMF leg receives the correct flow rate. Using multiple VMFs would be redundant, producing an over-specified system. As a consequence of continuity, the precise location of the VMF is essentially immaterial for this geometry. Fig. 3 shows the two VMF configurations used in this analysis. 


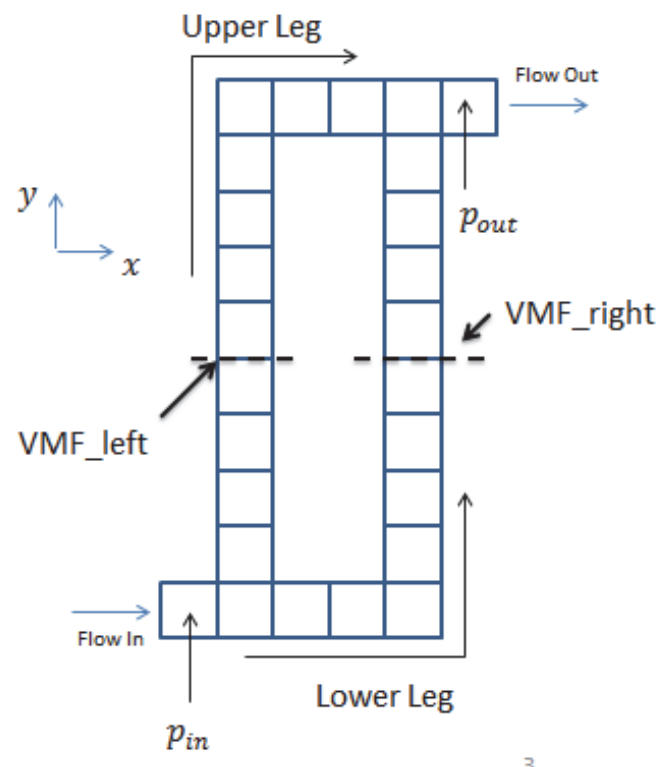

Fig. 3. Schematic diagram of Cartesian flow splitter test case with two potential VMF locations. VMF_left and VMF_right are only activated separately. The upper left and lower right corners are geometry-dictated stream direction changes. The stream direction changes at the lower left and upper right corners are dictated by pressure balance.

\subsubsection{Steady State}

For steady state verification, the inlet mass flow rate through the flow splitter was varied in the range $\min \in(\mathrm{Ogg}, 15.0 \mathrm{~kg} / \mathrm{s}]$. Mass flow rates through each loop are shown in Fig. 4 and total pressure drops are shown in Fig. 5. Coupled results were calculated with the IDO method. TRACE assumes fullydeveloped flow throughout the flow domain, while STAR-CCM+ calculates specific velocity profiles throughout. Based on this, TRACE would be expected to under-predict the pressure drop, but instead TRACE over-predicts the total splitter pressure drop as well as the amount of flow through the lower leg of the flow splitter. Evidently, in this case TRACE over-estimates the pressure penalty incurred by diverting flow and is more "reluctant" to change flow direction when not bound by geometry. Pressure drops resulting from flow changes are both distributed and multi-dimensional, and can only be approximated by the TRACE formulation. The coupled results collapse directly onto the STAR-CCM+ results, simultaneously confirming both the friction factor coupling and the VMF implementation. No differences are found between the VMF_left and VMF_right cases, confirming that the Newtonian iteration of Eqs. 5 through 7 has not been unduly disrupted and that the particular selection of VMF does not affect the coupled solution in this case. 

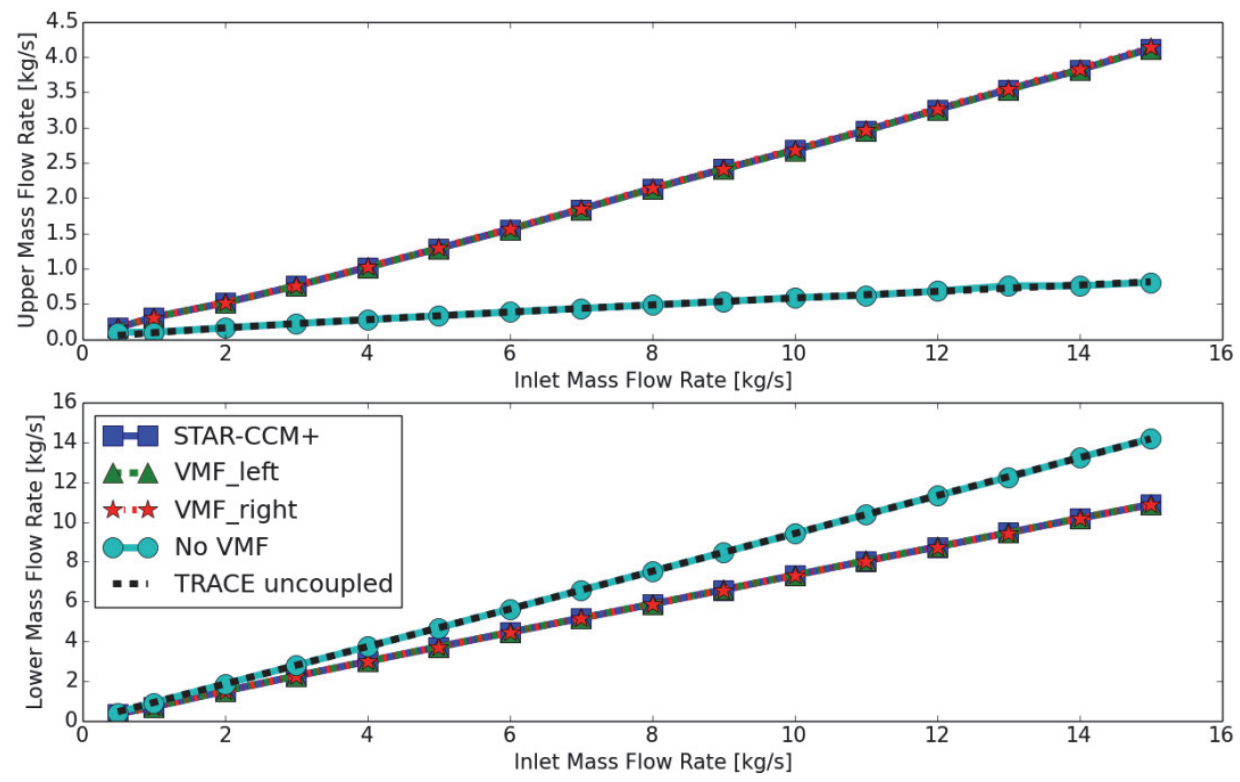

Fig. 4. Mass flow rates through each leg of the Cartesian flow splitter.

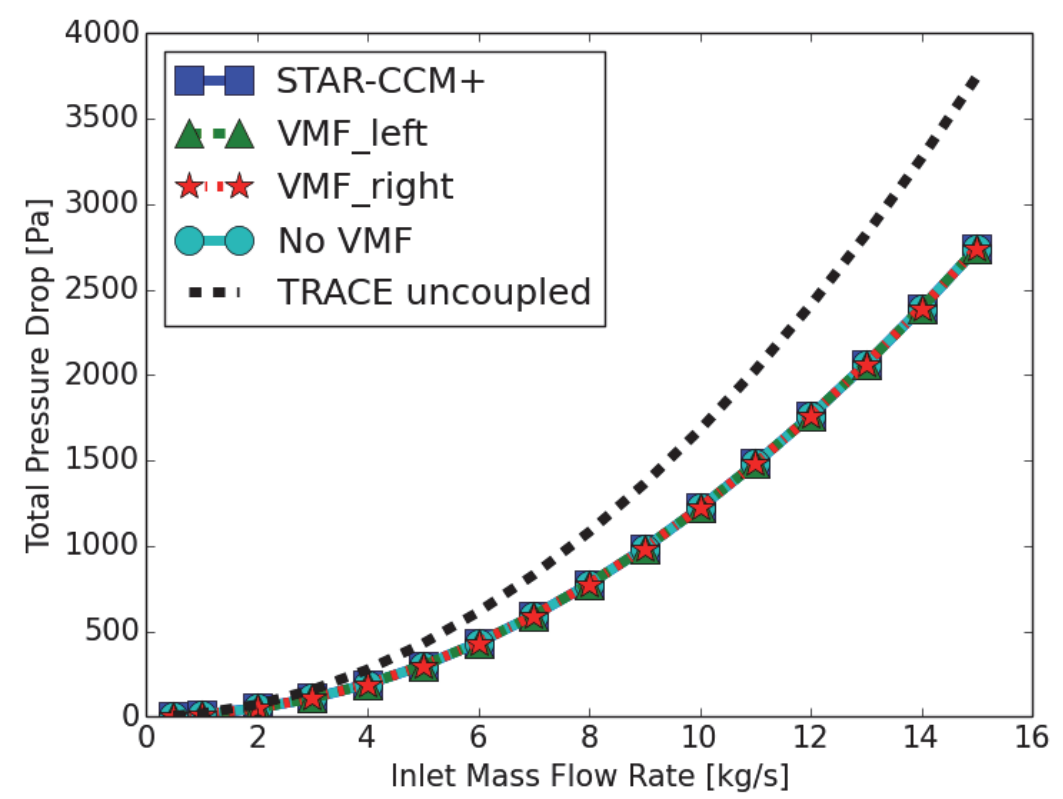

Fig. 5. Steady state global pressure drops across Cartesian flow splitter.

The behavior of the simulations performed with coupled friction factors, but without any VMFs, ("No VMF" in Fig. 4 and Fig. 5) is also of note. As evidenced in Fig. 4, the "No VMF" case produces the same mass flow distributions as TRACE standalone, while Fig. 5 shows that this case produces the same total pressure drop of STAR-CCM+. This result is clearly erroneous. Because the coupling algorithm allows TRACE to initialize unperturbed, the STH begins the coupled iteration with a standalone TRACE solution. Once CFD friction factors are passed to TRACE, the pressure drop in each leg is forced to match that of STAR-CCM+. Because the pressure-velocity feedback has effectively been disabled in the process, the STH solver does not change the velocities through the legs to match the pressure balance and hence the coupled solution produces the velocity field of standalone TRACE. This demonstrates that VMF is necessary to make sure that a consistent velocity-pressure field is computed by the coupled code. 


\subsection{Transient}

A time-dependent mass flow rate, given by Eq. 26, was applied to the inlet of the flow splitter to verify the IDO method in transient conditions:

$\operatorname{mint}=5.0 \mathrm{kgs}, \quad t \leq 150.0 \mathrm{~s}, 5.0 \mathrm{kgs} \times 1.0+\sin 4 t-150.0 \mathrm{~s} 10.0 \mathrm{~s} \pi, \quad 150.0 \mathrm{~s}<t<160.0 \mathrm{~s}, 5.0 \mathrm{kgs}, \quad 26$
$t \geq 160.0 \mathrm{~s}$.

Converged solutions for the system-wide pressure drop are shown in Fig. 6, while the accompanying mass flow rate distributions are shown in Fig. 7.

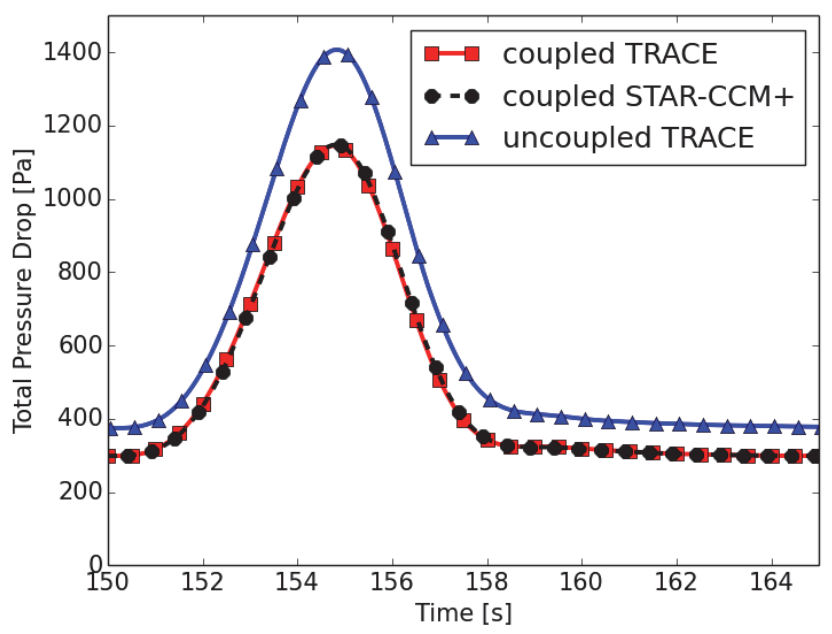

Fig. 6. Total system pressure drop for coupled and standalone simulations of the flow splitter transient.

TRACE over-predicts the total pressure drop and the flow rate through the lower leg throughout the transient, consistent with steady state observations. Additionally the upper mass flow rate in the uncoupled TRACE solution drops below zero, indicating recirculating flow, which is not predicted by STAR-CCM+. For both the pressure drop and mass flow distributions, the coupled TRACE solution matches the STAR-CCM+ solution very well. 

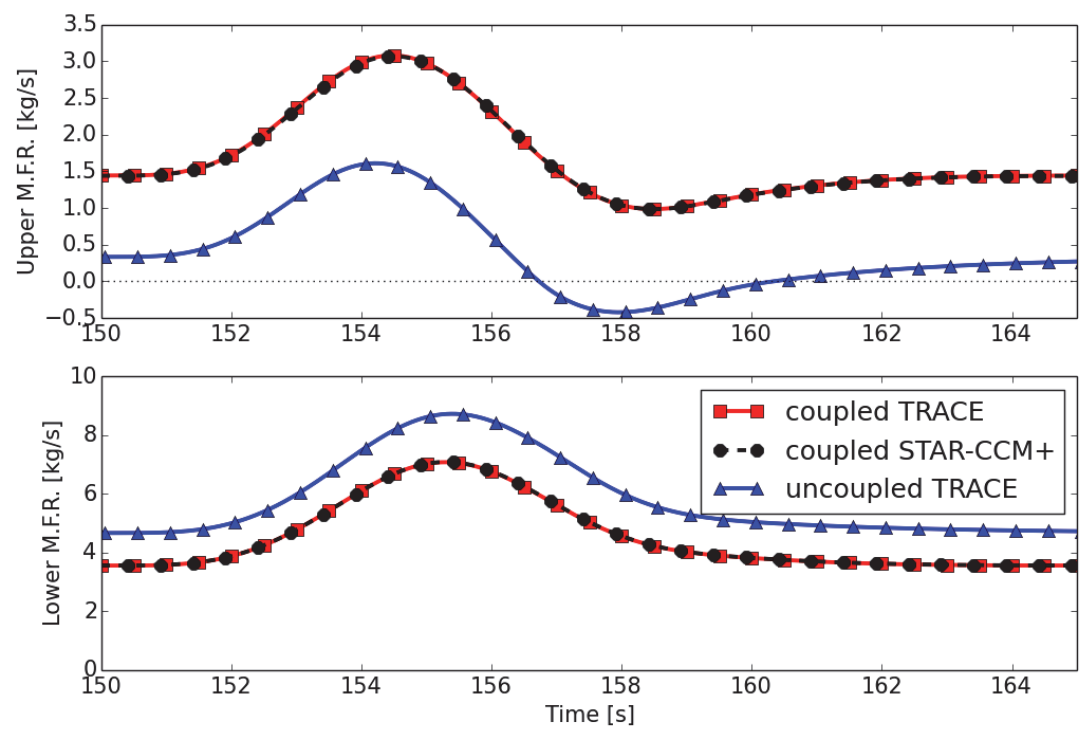

Fig. 7. Mass flow rates through the upper (top) and lower (bottom) legs of the flow splitter geometry under transient conditions.

The mass flow rate through the upper leg and the total pressure drop are shown in Fig. 8 for three selected integration time steps. The plots show that the coupling is not exact, due to the explicit nature of the numerical coupling, and the two codes exhibit some disagreement at larger time steps. The $\infty$-norm error, calculated with Eq. 27, is plotted in Fig. 9:

$$
E r r=\max 150 s \leq t \leq 170 s \Delta p T R C t-\Delta p C C M t \max 150 s \leq t \leq 170 s \Delta p C C M t .
$$

The error in the system pressure drop agreement levels off below 1\% around an integration time step of $\Delta t=0.01 \mathrm{~s}$. The error in mass flow rate agreement is relatively large for large time steps, but decreases steadily with time step. 

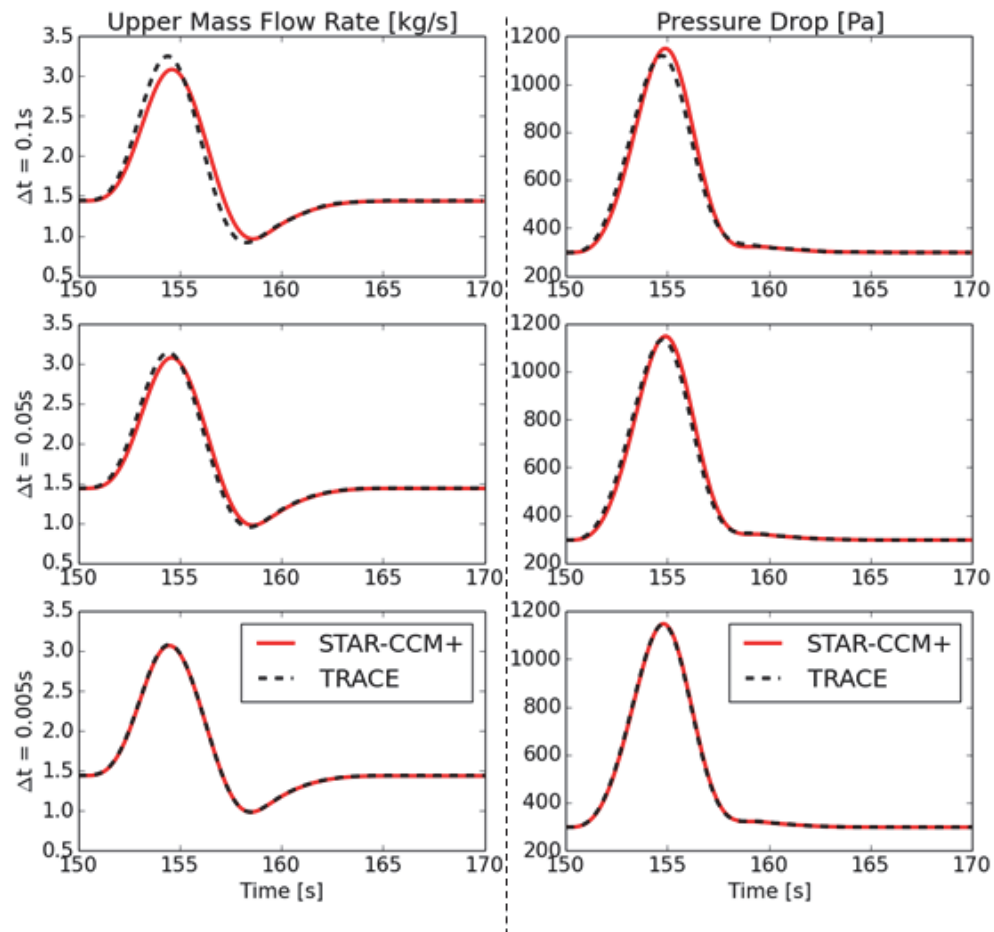

Fig. 8. Flow rate through upper leg (left) and system wide pressure drop (right) for the flow splitter test case. Both STAR-CCM+ and TRACE data sets are from coupled simulations.

Since the IDO method is used, no corrective action is taken for the CFD velocity applied to VMFs for this test case, and some additional error is introduced. Using the binomial theorem, the error can be estimated by as shown in Eq. 28:

$$
\begin{array}{cc}
V j+12 n+1-V C F D=\Delta P n+1-\Delta P n \Delta t \rho \Delta s \alpha 1+2 \Delta t K j+12 n V j+12 n=\Delta P n+1-\Delta P n \rho \Delta s \alpha \Delta t 1-2 \Delta t K j+12 n V j+12 n & 2 \\
+\ldots . & 8
\end{array}
$$

The predicted linear behavior is approximately observed in Fig. 9. 


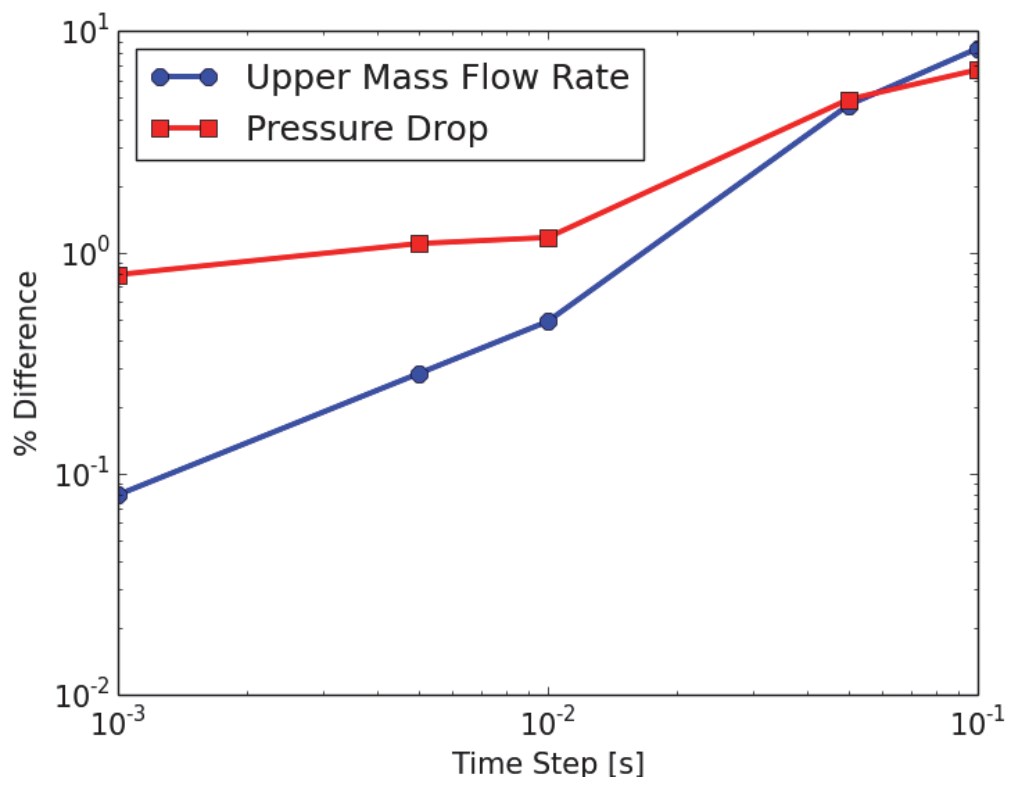

Fig. 9. $L \infty$ error $[\%]$ for upper mass flow rate and system wide pressure drop for the flow splitter test case.

\section{Annular Flow Splitter Verification Case}

The Cartesian geometry of the previous section presented ideal conditions for introducing VMFs and multi-dimensional coupling, but the well-defined flow paths provided significant simplification. In order to expand to geometries more representative of NPPs, an annular test case was devised. Dimensions of the base annulus are shown in Fig. 10 with overlaid TRACE nodalization. Flow enters the domain through a pipe attached to the third quadrant (Q3 in Fig. 10) on the highest axial level (see Fig. 10 right) and exits through the first quadrant (Q1) on the lowest axial level.
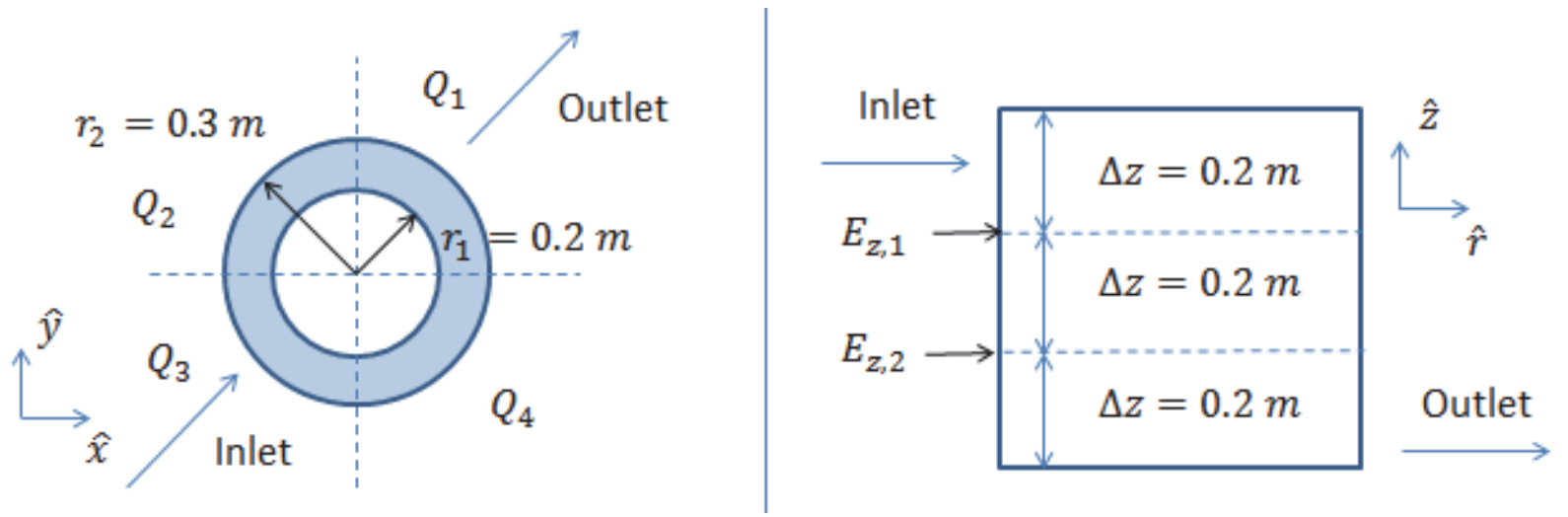

Fig. 10. Schematic diagram of annular geometry. (LEFT) Top down view showing annular rings divided into 4 azimuthal quadrants. (RIGHT) side view showing axial dimensions divided into 3 segments of equal length; labeled edges $(E z, i)$ also shown.

The corresponding TRACE and STAR-CCM+ meshes are shown in Fig. 11. The CFD mesh is composed of 173,022 cells, mostly polyhedral in nature with 2 layers of prismatic cells near wall boundaries. The inlet is made up of a mass flow boundary while the outlet consists of a pressure boundary. A standard $k-\epsilon$ turbulence model is used to satisfy closure. While the CFD mesh is relatively coarse, the purpose of this 
work is to verify the coupling method and to explore numerical behaviors. We seek only to demonstrate that a STH code can be corrected to produce CFD-like results in a coupled configuration. In this case, minimizing computational time is prioritized over physical accuracy. Once the coupling method is fully established and verified, a fully validated CFD model is required in order for results to be trustworthy.

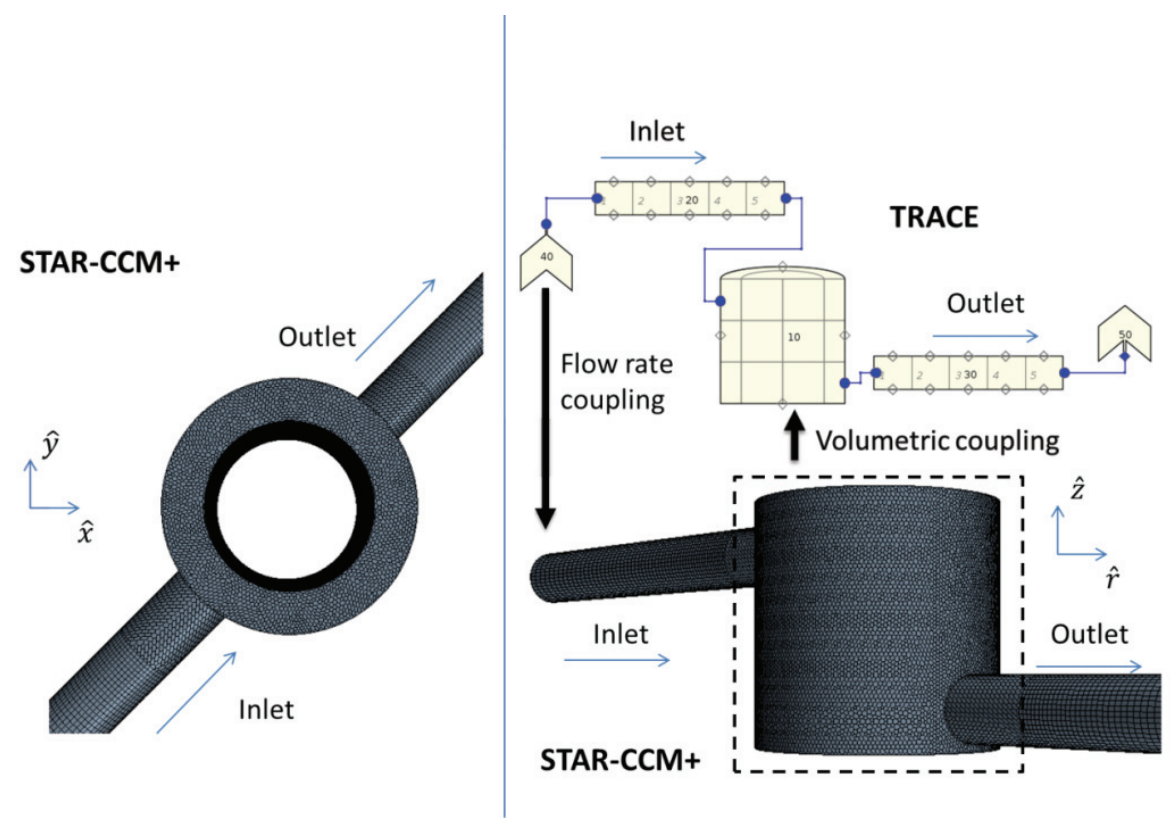

Fig. 11. Views of TRACE and STAR-CCM+ implementations of the annulus geometry. (LEFT) Top down view of CFD mesh. (TOP RIGHT) TRACE nodalization as displayed by the Symbolic Nuclear Analysis Package (SNAP). (BOTTOM RIGHT) side view of CFD mesh showing axial locations of inlet and outlet channels.

The coupling for this test case was realized through 2 interfaces, as indicated on the right side of Fig. 11. A surface interface passes mass flow rate data from a TRACE "fill" component to a STAR-CCM+ mass flow inlet. Additionally, a volumetric interface passes friction factor and velocity data from the STAR$\mathrm{CCM}+$ region to the TRACE "vessel" component. With both interfaces working together, the coupling is two-way.

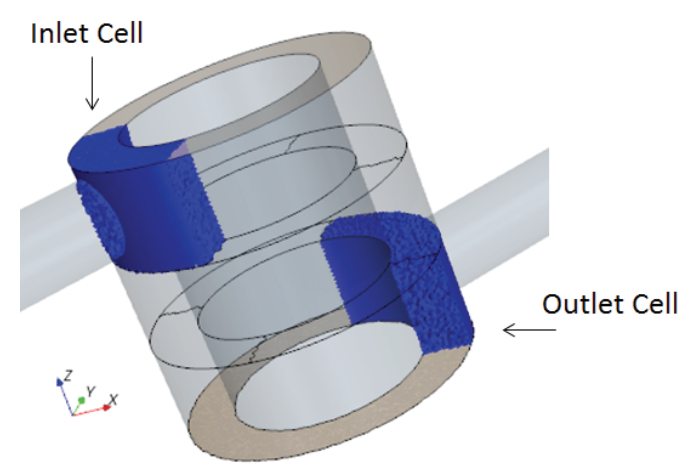

Fig. 12. Highlighted inlet/outlet cells showing definition of "global" pressure drop.

A set of pressure drops is defined to quantify the accuracy of the coupling with respect to the pressure field. Each pressure drop is defined in STAR-CCM+ as the difference in volume-averaged pressure between two cells. The TRACE definition of a pressure drop is identical, but TRACE pressure values are natively defined as volume averages, so no averaging needs to be carried out. A global pressure drop is defined as the difference between the "Inlet Cell" and "Outlet Cell" as denoted in Fig. 12. In more 
complex cases, a global pressure drop must include the losses incurred by flowing into inlet cells and out of outlet cells, since there is an abrupt change in flow area at these locations. However, the goal of this chapter is the verification of coupling in 3-D geometry, and treatment of entry/exit effects is deferred to future work with closed loop flow configurations in which the local pressure drops have a substantial effect on the system.

It is secondarily desired to characterize the behavior of the coupling on a more localized scale. At each edge, or boundary between adjacent cells, a velocity is defined in TRACE. Additionally, a pressure drop can easily be defined at the edge as the difference in volume-averaged pressure between the adjacent cells. As shown in Fig. 10, the annulus has 8 internal axial edges ( 2 edges per quadrant with 4 quadrants) and 12 internal azimuthal edges ( 3 faces per azimuthal division with 4 divisions) for a total of 20 internal edges. The quality of coupled solutions is therefore based on a combination of global and local pressure drops, along with local velocity profiles.

In order to quantify the fidelity of the coupling with respect to the pressure field, the error functions of Eqs. 29 and 30 are defined. Because of the explicit time stepping between the CFD and STH code data exchange, the STAR-CCM+ curves are shifted by one time step. This has minimal effect on the value of Err 2, but it allows Erro to produce more useful values, as will be demonstrated. All transients tested in this chapter are driven by inlet mass flow rates with time dependence that begins, arbitrarily, at $t=75.0 \mathrm{~s}$ and ends at $t=80.0 \mathrm{~s}$. The error function integrates the pressure drop functions beginning $3.0 \mathrm{~s}$ before and after these times in order to capture redistribution effects. Eq. 29, based on the 2-norm, can be interpreted as the time-averaged difference between the CFD and STH pressure drops:

$$
\operatorname{Err} 2=111.0 s t=72.0 s t=83.0 s \Delta p T R C t^{\prime}-\Delta p C C M t^{\prime}+\Delta t d t^{\prime},
$$

while Eq. 30, based on the $\infty$-norm, is interpreted as the maximum value of the difference over the course of the transient:

$$
\operatorname{Err} \infty=\max 72.0 s \leq t \leq 83.0 s \Delta p T R C t-\Delta p C C M t+\Delta t .
$$

Analysis of both types of error will provide a better picture of convergence for transients with, for instance, abrupt changes in the time derivative of the total flow rate through the system, as will be encountered in $\$ 4.3 .1$ and $\S 4.4 .1$. Convergence in the sense of Erro is a stronger statement than convergence in the sense of Err2.

A similar approach is adopted for the velocity field, although an $\infty$-norm error function was not deemed necessary due to smoothness of the velocity fields encountered in this paper. Eq. 31 is employed to quantify error in the velocity field on a global scale:

$$
\text { Errvel }=i=120111.0 \mathrm{st}=72.0 \mathrm{st}=83.0 \mathrm{svi}, T R C \mathrm{t}^{\prime}-v i, \mathrm{ccmt}{ }^{\prime}+\Delta t d t^{\prime} .
$$

The summation ranges over all faces in the system, both velocity-matched and open. With units of velocity, this quantity has the interpretation of the time-averaged total difference in velocity between TRACE and STAR-CCM+. While dividing by 20 would yield the average error per face, it is found in practice that the majority of the error is confined to specific faces, causing such an interpretation to be misleading. 


\subsection{Velocity Matching Face Configurations}

In the Cartesian case of $\S 3$, the placement of VMFs was relatively straightforward, due to the existence of two distinct flow paths. In true reactor geometry, flow enters an annular downcomer primarily travelling downward, then makes a turn in the lower plenum and finally rises through the core and riser. Although the geometry of this section was designed to represent a downcomer, the configuration of the inlet and outlet create much more azimuthal flow than would be expected in a NPP. The VMF configurations devised for this case were based on decomposing the geometry into 4 "pseudo flow paths" corresponding with the 4 azimuthal sections of the annulus. Each path therefore consists of two axial pressure drops. This VMF scheme was chosen because the authors expect such a strategy to be directly extensible to more realistic NPP geometries. We applied VMFs to the axial faces along these paths for 3 of the 4 pseudo flow paths as shown in Fig. 13 (left). This allows the TRACE continuity equation to determine the flow through the $4^{\text {th }}$ face, preventing an overdetermined system. In this configuration, the azimuthal direction is entirely left to the TRACE continuity solver.

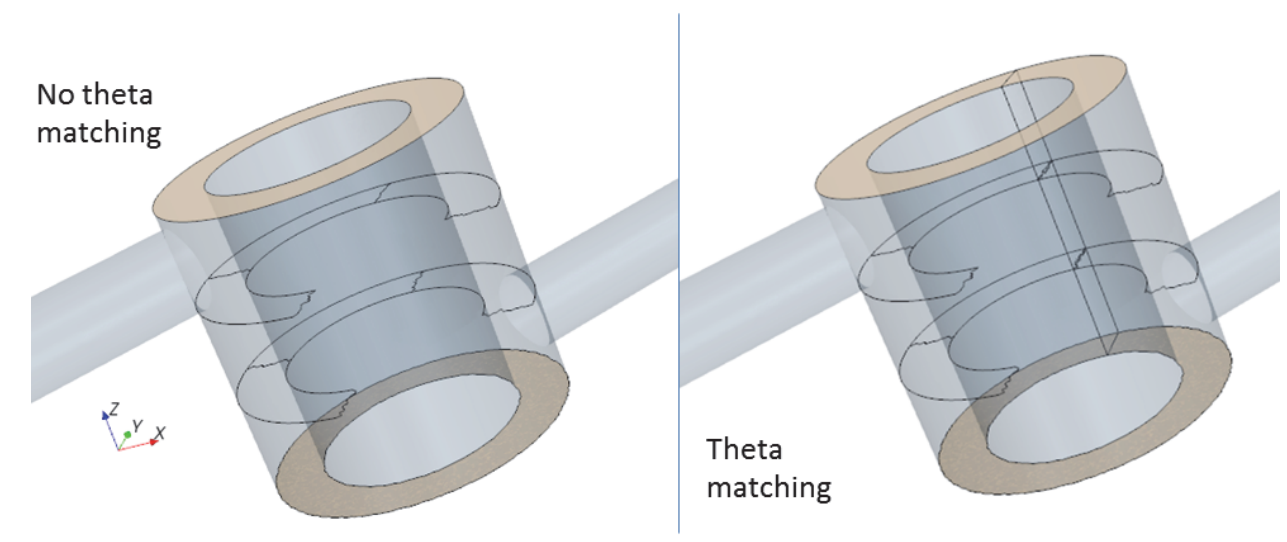

Fig. 13. Diagram showing VMF (outlined) placement for annular geometry. (LEFT) only axial matching faces. (RIGHT) theta matching faces included

In the VMF configuration shown in Fig. 13 (right), the three azimuthal faces of one sector are also included in the VMF configuration. In broad terms, one of the ultimate goals of this coupling methodology is to ensure proper velocity profiles at the core inlet and correct total pressure drops throughout the downcomer and inlet plenum regions. Correct matching of azimuthal velocities is not necessarily required to achieve this goal. As such, we included both cases to understand the effect that granting full control over azimuthal velocity to TRACE would have on stability and accuracy. The authors believe that study of these two configurations will lay the groundwork for a reasonable process for determination of VMF placement for reactor-like geometries.

\subsection{Steady State}

In order to provide a general idea of how the annular system behaves and how TRACE and STAR-CCM+ solutions differ, a rudimentary steady state analysis is performed. The inlet mass flow rate was varied with the resulting pressure drops across each axial edge (as indicated in Fig. 10) recorded as output. The data shown in Fig. 14 demonstrate that while TRACE standalone pressure drops behave similarly to those of STAR-CCM+, the magnitude of the pressure drops differ substantially. The coupled results, however, overlap the CFD results, verifying the correctness of the steady-state coupling. The inclusion of $\theta$ matching faces was found to have no effect and Fig. 14 results are representative of both VMF configurations shown in Fig. 13. 

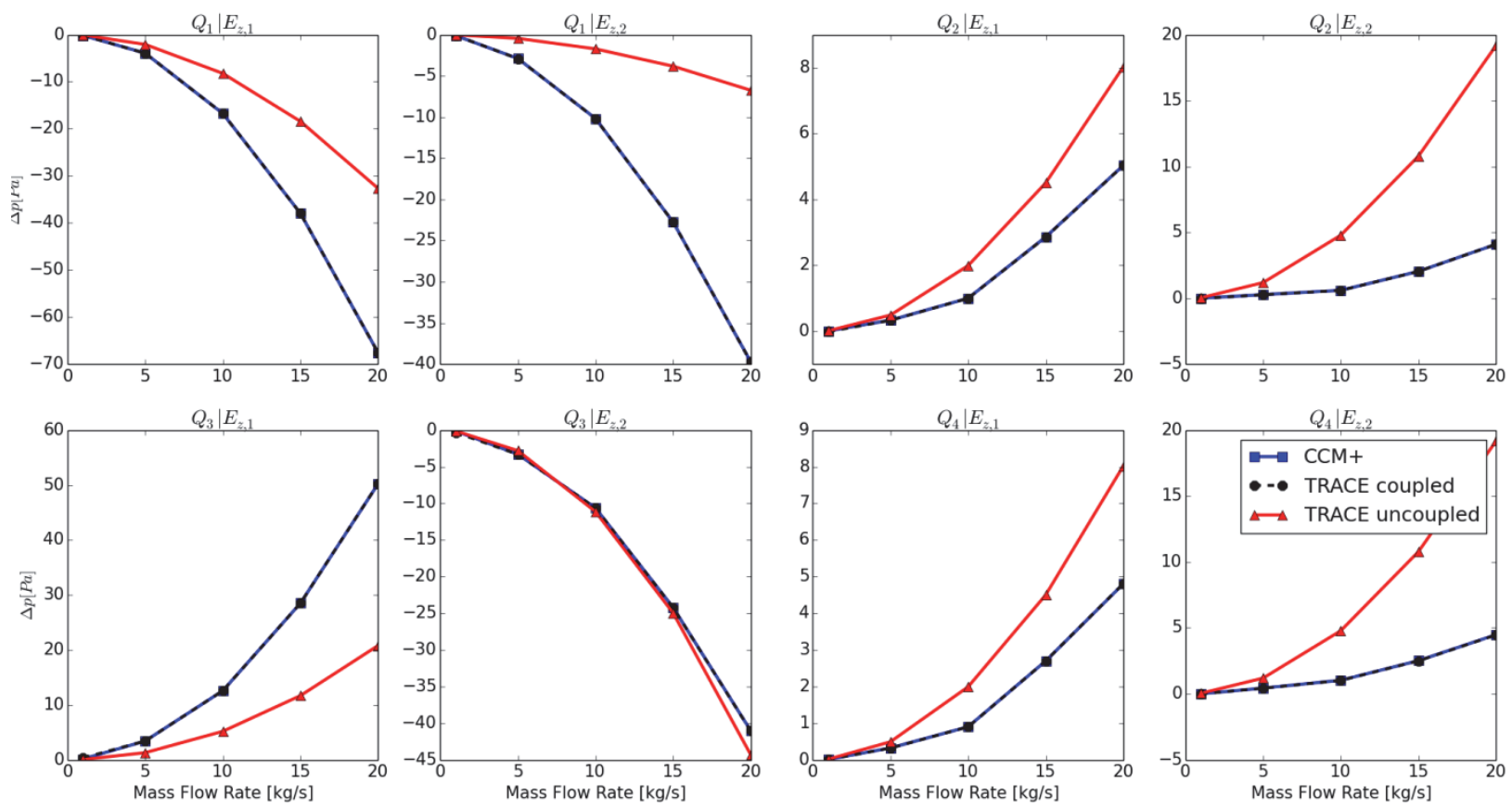

Fig. 14. Steady state pressure drop across each axial face for the annular test case.

The velocity field behaved similarly to the pressure field in that the uncoupled TRACE results differed noticeably from the CFD results, but the coupled version of TRACE was found to overlap STAR-CCM+ quite closely. In contrast to the pressure field, the velocity field does depend somewhat on the VMF configuration. Cases without $\theta$-matching can see different flows in the azimuthal direction. This behavior will be discussed in $\S 4.3 .1$ and is not shown here to avoid undue redundancy.

\subsection{High Flow Transient}

Coupling performance in transient mode is additionally sensitive to the rates of change of flow, rather than just the flow rate itself, as in steady state. In order to be sufficiently broad in our analysis, we executed simulations with three types of transient inlet mass flow rates in a high and a low flow regime respectively. This section discusses the "high flow regime" in which the initial mass flow rate is 15.0 $\mathrm{kg} / \mathrm{s}$, while a "low flow regime" is discussed in $\S 4.4$. Higher flow rates provide a greater stabilizing influence of frictional loss compared to the potentially destabilizing effect of the inertial component of the pressure gradient. The ratio of these two effects is the Reynolds number, which takes on a value in the range $R e \in 1.28 \cdot 104,1.95 \cdot 105$ for each face at the initial mass flow rate. Transients in this class are therefore considered to be less challenging than those of $\S 4$.4. The specific transients considered in this section are driven by the time dependent inlet mass flow rates shown in Fig. 15 (TOP). The time derivatives of the same functions are shown in Fig. 15 (BOTTOM). The "linear" test case is characterized by a mass flow rate that increases proportionally with time during the transient. The "sin" ${ }^{4}$ " functions are both based on quartic sinusoids designed to have continuous first-order time derivatives. The quartic sinusoids are further classified by the relative size of their first order time derivatives. The function with the higher value derivative is referred to as "fast" or "tall", while the function with the lower value derivative is referred to as "slow" or "short." As can be seen in Fig. 15, the time derivative of the tall quartic sinusoid reaches a rather high value compared to the other two functions, which have similar maxima. 

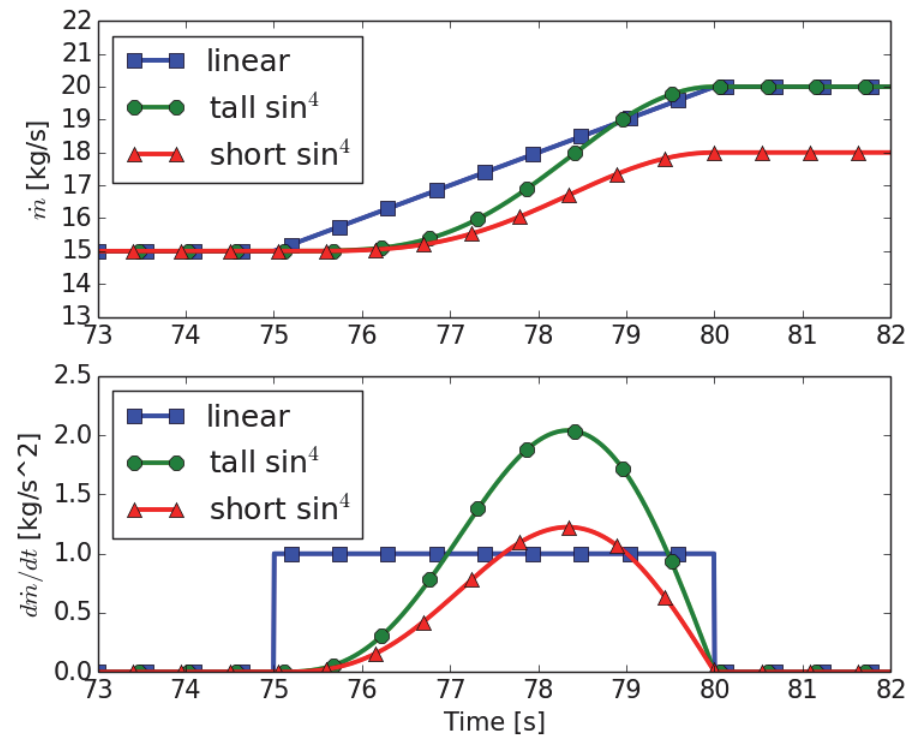

Fig. 15. (TOP) Time dependent mass flow rates used for transient test cases. (BOTTOM) derivative w.r.t. time for the transient mass flow rates.

\subsubsection{Linear Mass Flow Rate}

For the linear mass flow rate in the high flow regime, the time dependent mass flow rate of Eq. 32 is applied to the flow inlet

$$
\begin{aligned}
\operatorname{mint}= & 15.0 \mathrm{kgs}, t \leq 75.0 \mathrm{~s}, 15.0 \mathrm{kgs}+t-75.0 \mathrm{skgs}, \\
& \text { 75.0s }<t<80.0 \mathrm{~s}, 20.0 \mathrm{kgs}, t \geq 80.0 \mathrm{~s} .
\end{aligned}
$$

As shown in Fig. 15, the defining features of this transient are the discontinuities in the time derivative of the mass flow rate at $t=75 \mathrm{~s}$ and $t=80 \mathrm{~s}$. The convergence pattern for the global pressure drop (from inlet to outlet as defined in Fig. 12) for this transient is shown in Fig. 16. A striking feature of these curves is the oscillatory behavior near $t=75 \mathrm{~s}$ and $t=80 \mathrm{~s}$. As the time step is refined, the decay ratio increases and the error decreases noticeably, especially away from the time derivative discontinuities. However, the oscillations near the discontinuities persist with seemingly constant amplitude for even very small time steps, similar to the familiar Gibbs phenomenon. 


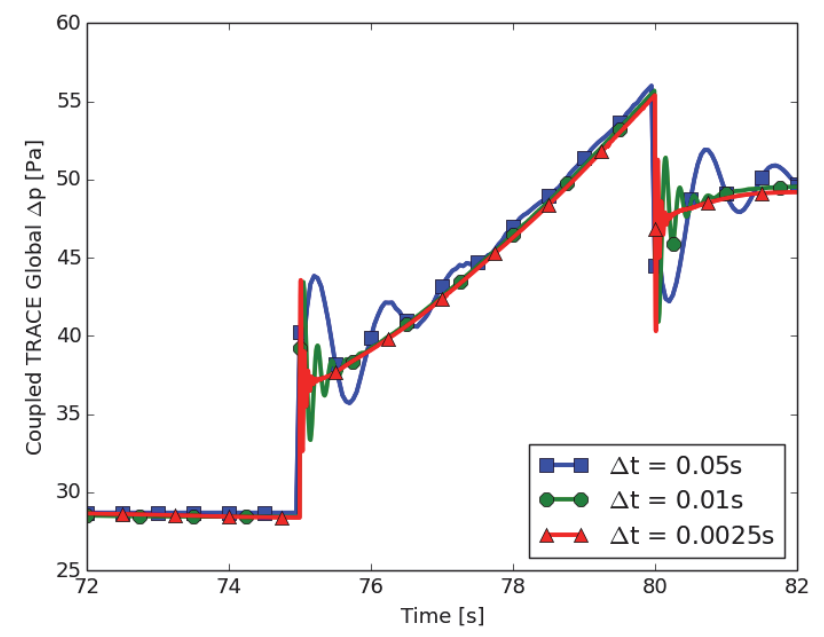

Fig. 16. Typical convergence pattern for global pressure drop subject to the linear mass flow rate in the high flow regime as computed with selected representative time steps.

The oscillatory response can be explained by examining VMFs in greater detail. Consider an explicit time stepping coupling configuration with $N V M F$ velocity-matched faces out of a total of $N$ faces subject to the transient of Eq. 32. During the time step in which the linearly increasing mass flow rate is first engaged (i.e. $t n=75.0 s, t n+1=75.0 s+\Delta t$ in TRACE), the total mass flow rate through the system increases by $\Delta m=\Delta t k g s 2$. Broadly approximated, in an uncoupled solution the additional mass flow rate will be split evenly among all $N$ edges of the system:

$$
\Delta m \text { face } \sim \Delta m N
$$

where $\Delta m f a c e$ is the amount the mass flow rate increases during the time step across each face. However, in a coupled case, the VMFs are still held at the velocities calculated with CFD at $\min =15.0 \mathrm{kgs}$, since the mass flow rate increase had not been communicated to CFD during the previous time step. The faces that are not velocity-matched must therefore compensate, resulting in an over-estimated inertial pressure that reverberates through the entire system. By continuity, a total flow rate $N V M F \triangle m f a c e$ needs to be made up by uncoupled faces. An open face flow rate given by Eq. 34 is calculated by assuming that the makeup flow rate is evenly distributed among the unmatched faces:

$$
\begin{gathered}
\Delta \text { mopen face } \triangle m N+N V M F \\
\Delta m \text { face } N-N V M F=\Delta m N N N-N V M F=C V M F \Delta m \text { face. }
\end{gathered}
$$

The accompanying inertial pressure drop can therefore be estimated by Eq. 35 which predicts an overprediction of the inertial pressure drop by a factor of $C V M F$ :

$$
\Delta p I \sim \Delta x \Delta t \Delta \text { mopen face } A \text { face }=C V M F \Delta x \Delta t \Delta m \text { face } A \text { face } .
$$

where $A$ face is the area of the face and $\Delta p I$ is the change in inertial pressure during the time step. For the case without $\theta$-matching, $N=20$ and $N V M F=6$, leading to $C V M F \approx 1.43$. Including azimuthal VMFs, $N V M F=9$ and $C V M F \approx 1.82$. While this analysis is extremely simplified, it predicts that the inertial 
pressure drop will be initially over-predicted by about $40 \%$ with $\theta$-matching and by about $80 \%$ without $\theta$ matching. These predictions match up well with the observations in Fig. 17, which shows the initial portion of the transient calculated with each coupling method for two selected time steps. Also apparent in Fig. 17 is the motivation for shifting the STAR-CCM+ results by one time step in Eqs. 29 and 30. The CFD data clearly lags the STH data by a time step, and the shifting effectively excludes the error that results from the lag. The value of Err 2 is only minimally affected, while the shift allows Erro to characterize the initial pressure overshoot rather than just the pressure of the first time step. Without the shift, Erro was found to characterize the time step lag rather than the inertial pressure overshoot.
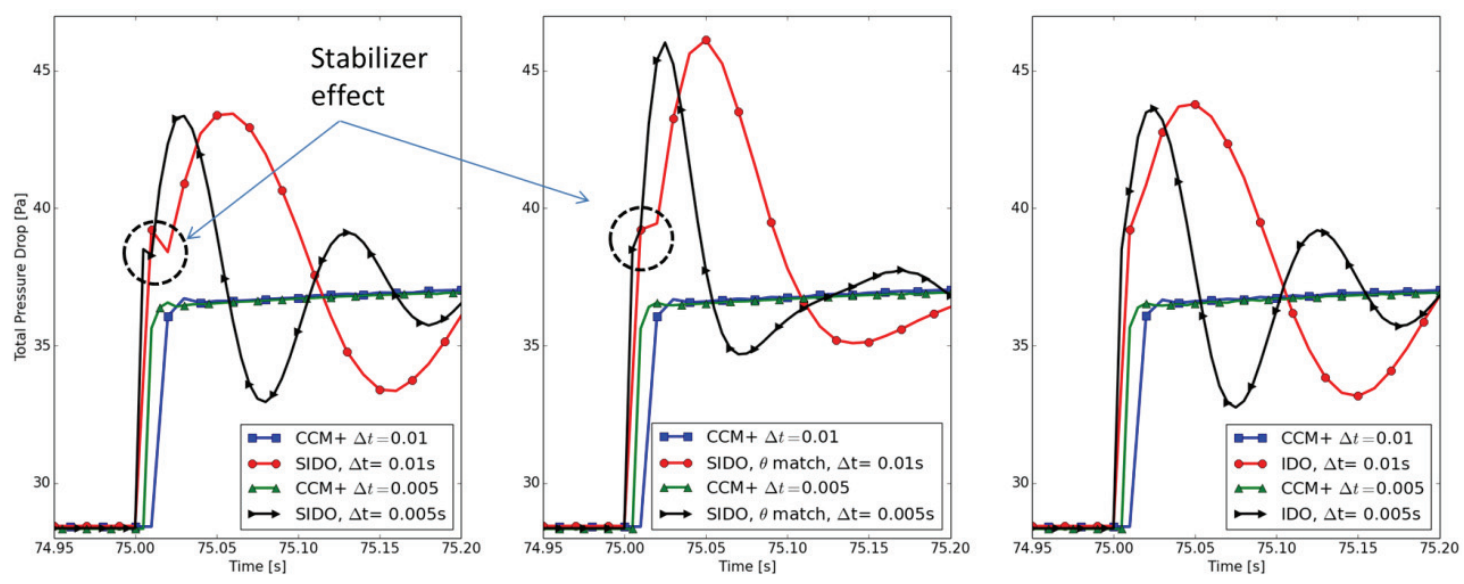

Fig. 17. Plots of coupling behavior near the beginning of the high flow linear transient. (LEFT) Case with friction factor and VMF correction. (CENTER) SIDO case with $\theta$-matching. (RIGHT)

Coupled case without friction factor or VMF correction. Red/blue curves calculated with $\Delta t=. s$. Black/green curves calculated with $\Delta t=. s$.

In the SIDO cases (left and middle), there is a clear error reduction effect in that the TRACE curves (black and red) exhibit an abrupt direction change toward the STAR-CCM+ curves (blue and green). This stabilizer effect is less striking in the $\theta$-matched case (middle) and absent from the IDO case (right). While the effect seems rather small in Fig. 17, the effect on the $\infty$-norm error is substantial, as can be observed in Fig. 18 (left), where the difference between the SIDO and IDO curves is readily apparent for all time steps. The SIDO method with $\theta$-matching resulted in the least 2 -norm (left) error at large time steps and the most error at small time steps. At small time steps, the SIDO method without $\theta$-matching and the IDO method converge to the same value. The $\theta$-matching leads to significantly higher error in the œ-norm (Fig. 18 right) as predicted by Eq. 35 . 

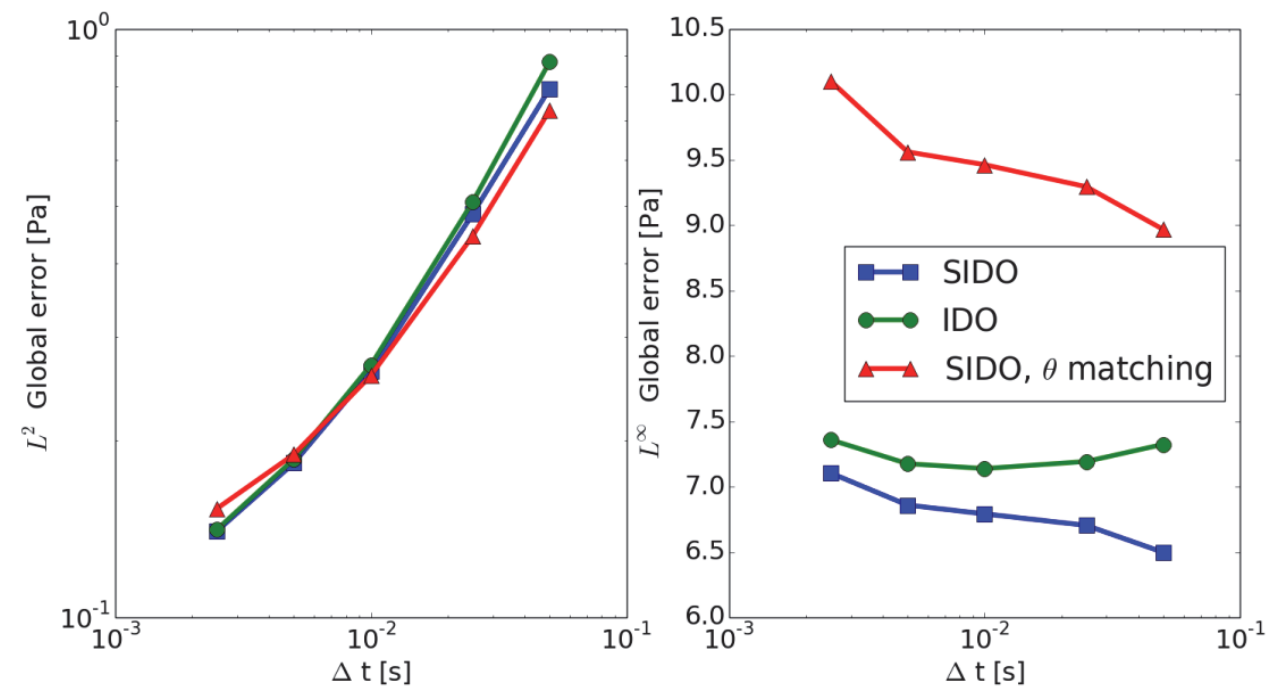

Fig. 18. Pressure convergence data for linear transient in the high flow rate regime. (LEFT) -norm error data. (RIGHT) $\infty$-norm error.

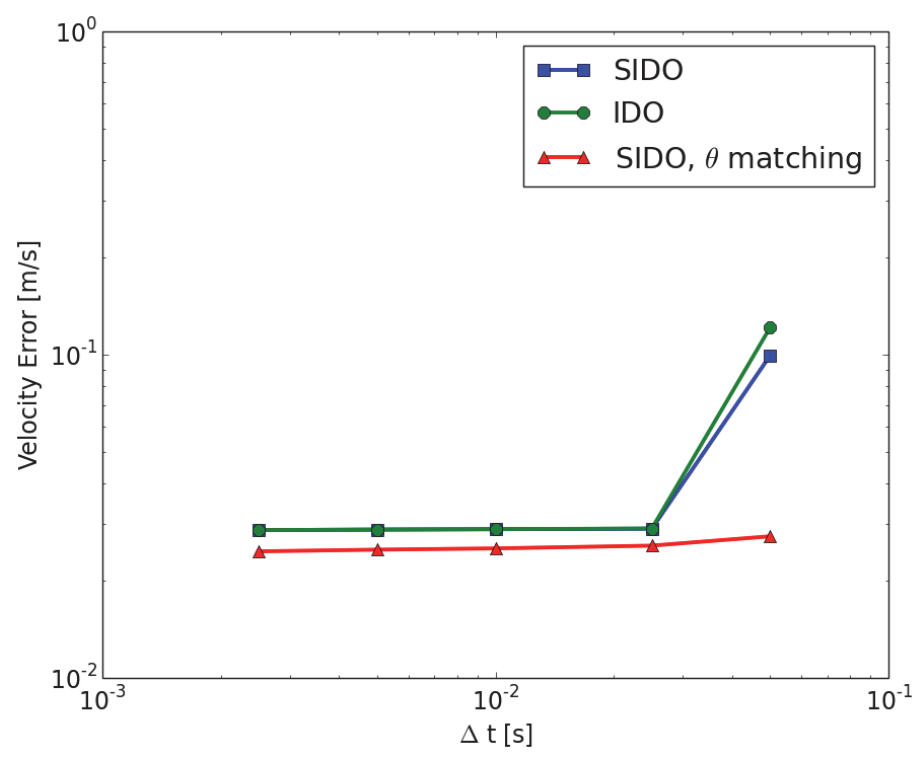

Fig. 19. Velocity convergence data for linear transient in high flow regime.

The velocity field error is shown Fig. 19 and is essentially constant, except for the large time step cases. The error is small but non-vanishing. As the time step is refined, the TRACE velocities on VMFs quickly match the STAR-CCM+ velocities, and the error reaches the ultimate value around $\Delta t \approx 0.025 \mathrm{~s}$. Unmatched faces are primarily responsible for the persistent error, as can be observed in Fig. 20. The figure shows the velocities across the 4 azimuthal faces on the lowest axial level of the annulus calculated with $\Delta t=0.05 \mathrm{~s}$. In the $\theta$-matched case, the $\theta 0 z O$ face is velocity-matched, while the other 3 faces are open. In the cases without $\theta$-matching, all 4 azimuthal faces are open. From these curves, it is apparent that open faces are the primary contributors to Errvel. The $\theta$-matched case shows significantly less error for all faces, while the SIDO case without $\theta$-matching appears to provide some error relief over an IDO case without $\theta$-matching. The data in Fig. 20 are indicative of the remainder of the faces in the system. In 
all cases, axial velocities are matched quite well due to the heavy velocity-matching in this direction. The primary goals of the coupling are to match overall system pressure drop and flow rate through important interfaces, such as the core inlet. The remainder of the TRACE solution is essentially discarded, so internal consistency between TRACE and STAR-CCM+ is not necessarily required.
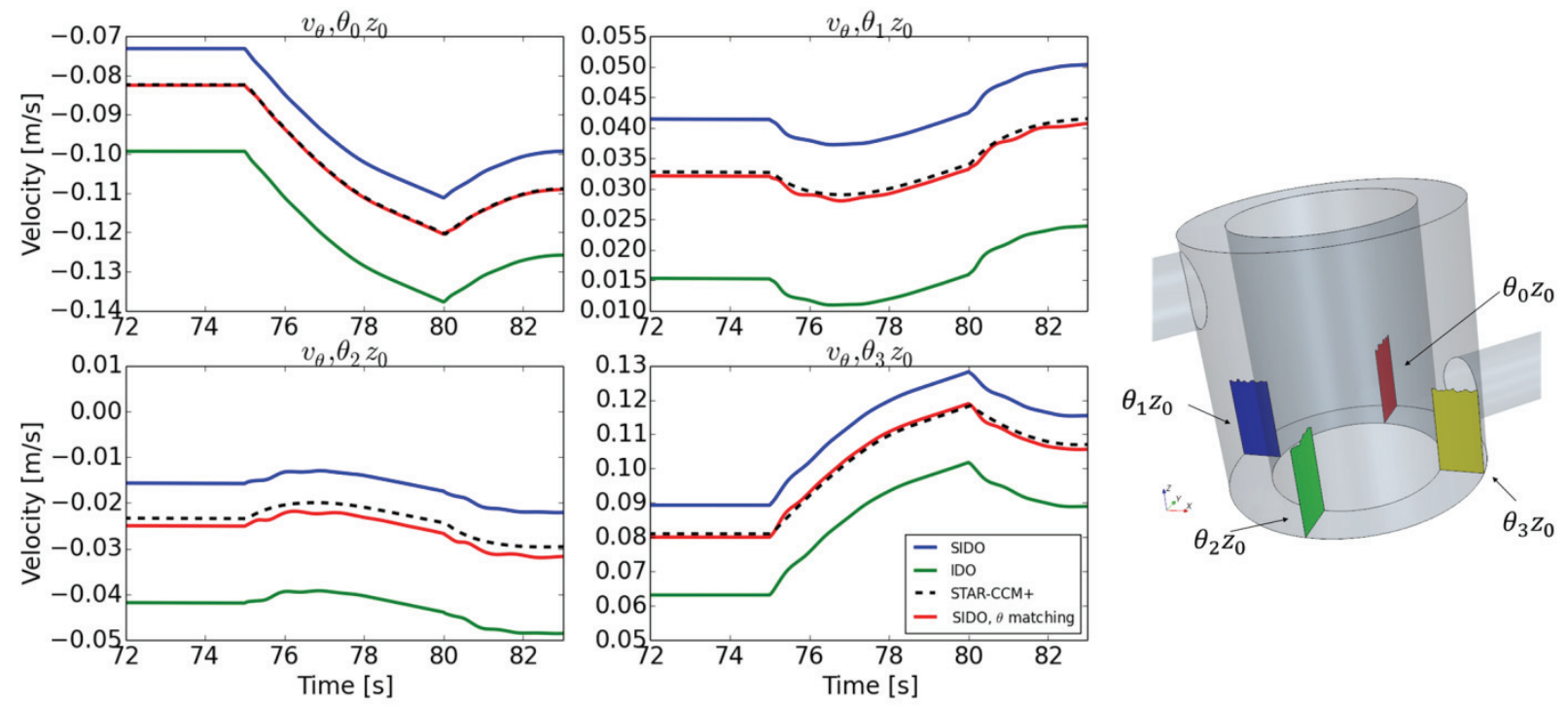

Fig. 20. (LEFT) Azimuthal velocities on the lowest axial level for the linear transient in the high flow regime. Calculated with $\Delta t=$. s. (RIGHT) Diagram showing location of VMF planes.

Coupled results for the SIDO method with and without $\theta$-matching and for IDO are shown in Fig. 21 compared to a reference standalone STAR-CCM+ curve. The curves are essentially indistinguishable from each other except near the discontinuities of the time derivative, at $t=75 \mathrm{~s}$ and $t=80 \mathrm{~s}$, verifying all employed coupling methods for this transient.

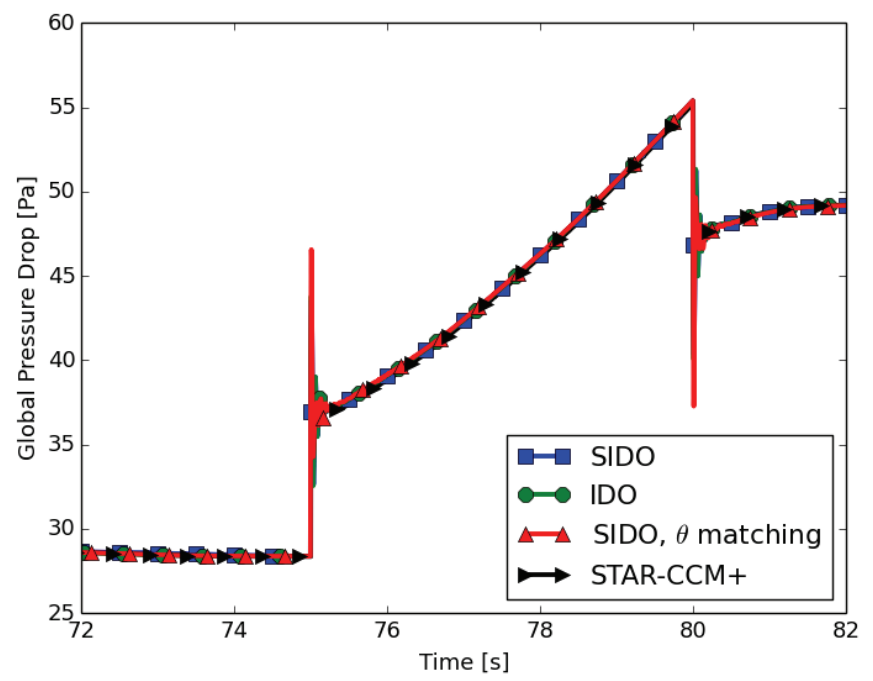

Fig. 21. Global pressure drop functions calculated with $\Delta t=. \quad s$ for the linear inlet mass flow rate in the high flow regime. 


\subsubsection{Fast Smooth Transient}

In the high flow regime, a fast, smooth transient is realized with a mass flow boundary condition set by Eq. 36. In contrast to the linear transient, this function presents no discontinuity in the inertial pressure drop:

$$
\begin{gathered}
\text { mint }=1 \text { 15.0kgs, } t \leq 75.0 \mathrm{~s}, 5.0 \mathrm{kgs} 1+\sin 4 t-75.0 \mathrm{~s} 10.0 \mathrm{~s} \pi, \\
75.0 \mathrm{~s}<t<80.0 \mathrm{~s}, 20.0 \mathrm{kgs}, t \geq 80.0 \mathrm{~s} .
\end{gathered}
$$

A typical convergence pattern for this class of transient is shown in Fig. 22. The solution does not appear to change significantly for time step refinements below $\Delta t=0.01 \mathrm{~s}$. The lower time step solutions appear to lag the higher time step solution, due to the combined effects of explicit time stepping and VMFs through Eq. 35.

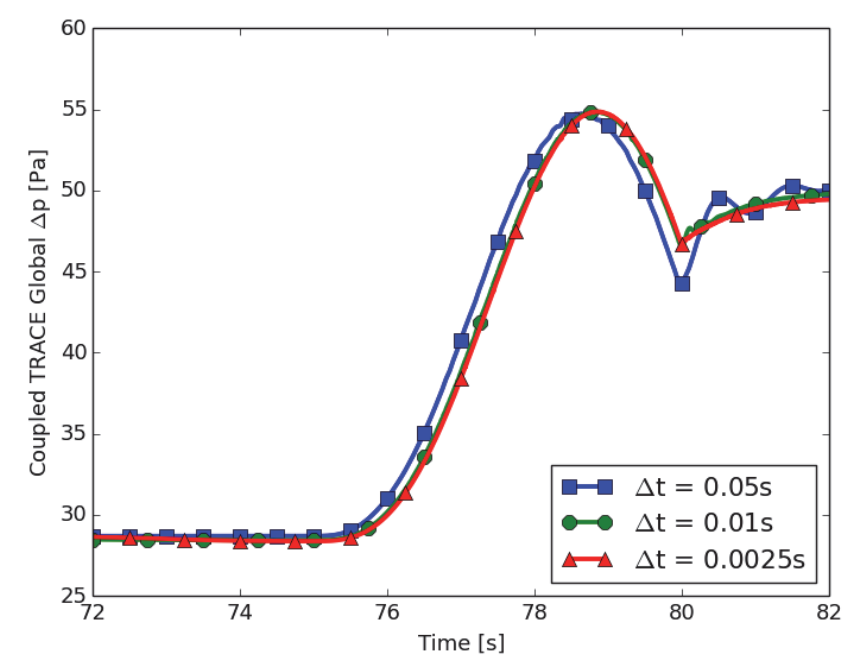

Fig. 22. Convergence pattern for fast smooth transient in the high flow regime. Results shown are as gathered from TRACE during a coupled simulation for selected time steps.

The pressure convergence behavior is shown in Fig. 23 calculated with both the $L 2$ (LEFT) and $L_{\infty}$ (RIGHT) norms. In this case the inclusion of the stabilizer term in the friction factor does not appear to have a substantial effect. The VMF-induced error described by Eq. 35 is apparent in these results, but is much less dramatic due to the smoother nature of the time derivative of the mass flow rate. The error in the velocity field, shown in Fig. 24, behaves quite similarly to the previous case in that the error quickly levels off to a constant value which results from unmatched faces. 

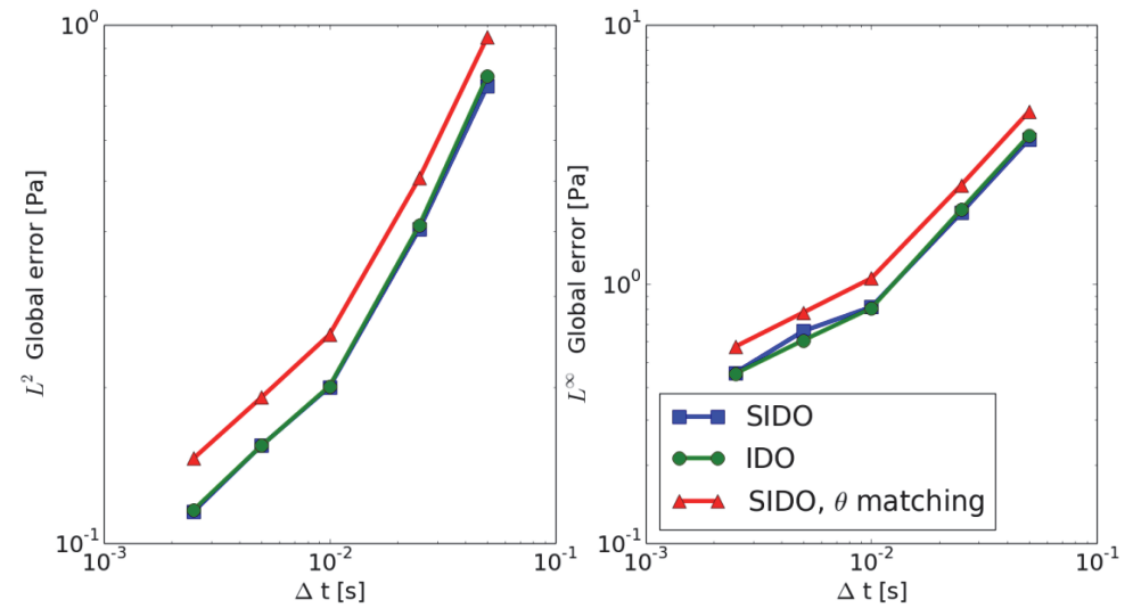

Fig. 23. Pressure convergence data for fast smooth transient in the high flow rate regime. (LEFT) norm error data. (RIGHT) $\infty$-norm error.

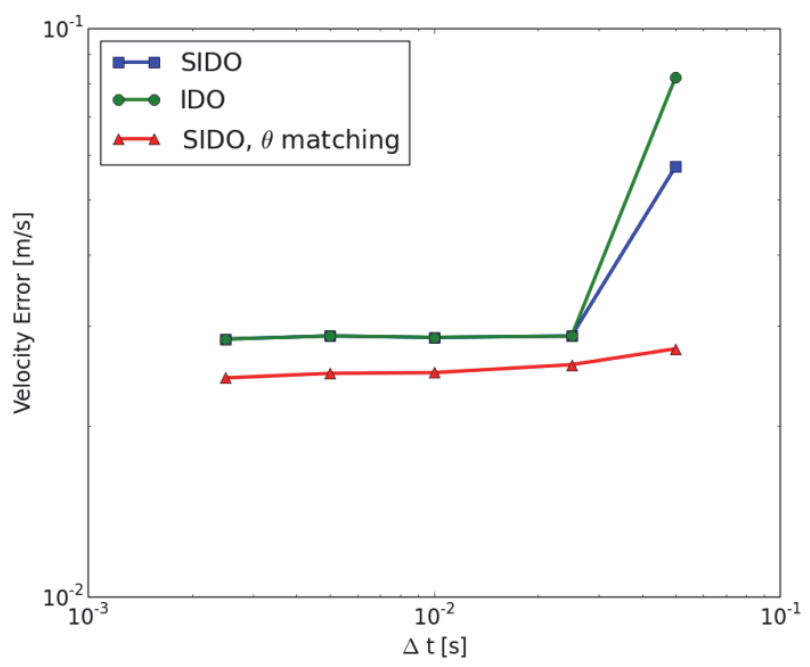

Fig. 24. Velocity convergence data for fast smooth transient in high flow regime.

Coupled results for the SIDO method with and without $\theta$-matching and for IDO are shown in Fig. 25 compared to a reference STAR-CCM+ curve. The curves are essentially indistinguishable throughout the transient, confirming the methods for this transient. Due to the smooth nature of the transient and the strong stabilizing effect of friction resulting from high flow rates, no oscillatory behavior is observed in the coupled solutions. 


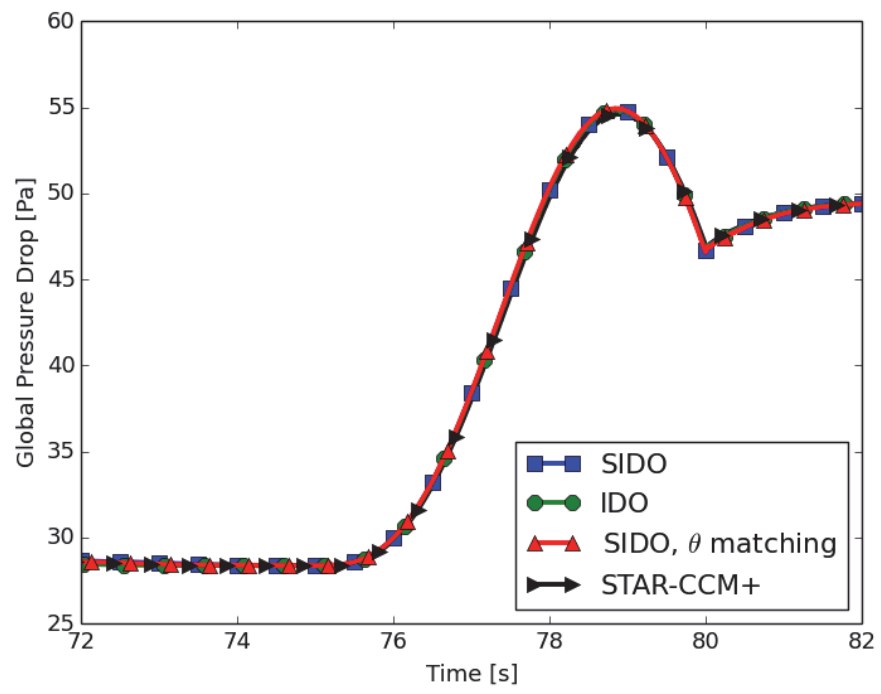

Fig. 25. Global pressure drop functions calculated with $\Delta t=. \quad s$ for the fast smooth inlet mass flow rate in the high flow regime.

\subsubsection{Slow Smooth Transient}

In the high flow regime, a slow smooth transient is realized through a mass flow boundary condition set by Eq. 37. The mass flow function has the same form as the in the fast smooth transient of the previous section, but it reaches a lower maximum value over the same time period and hence has a lower time derivative:

$$
\begin{gathered}
\operatorname{mint}=15.0 \mathrm{kgs}, t \leq 75.0 \mathrm{~s}, 15.0 \mathrm{kgs} 1+35 \mathrm{sin} 4 t-75.0 \mathrm{~s} 10.0 \mathrm{~s} \pi, \\
75.0 \mathrm{~s}<t<80.0 \mathrm{~s}, 18.0 \mathrm{kgs}, t \geq 80.0 \mathrm{~s} .
\end{gathered}
$$

Under higher flow conditions, the slow smooth transient does not behave appreciably different than the fast smooth transient. The effects of stabilizer corrections on friction factors and VMFs do not have a strong effect in either the 2-norm or the $\infty$-norm, as shown by the similarity of the IDO and SIDO method in Fig. 26. The velocity field error in Fig. 27 shows a leveling off of error around $\Delta t=0.025$ s. 

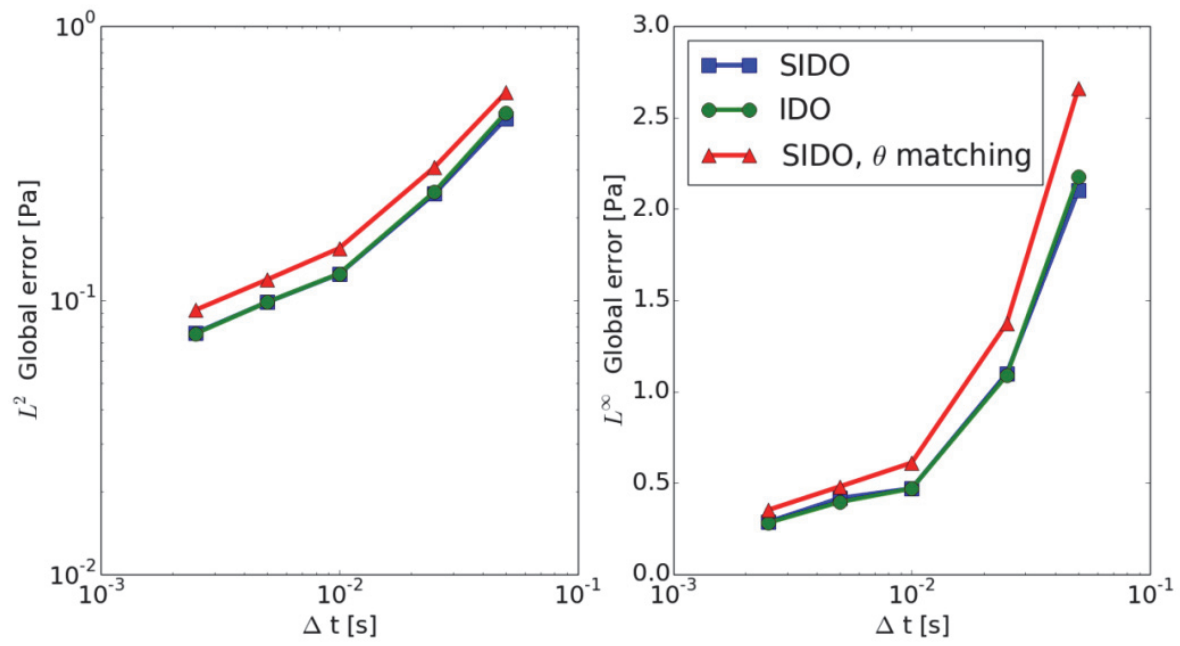

Fig. 26. Pressure convergence data for slow smooth transient in the high flow rate regime. (LEFT) -norm error data. (RIGHT) $\infty$-norm error.

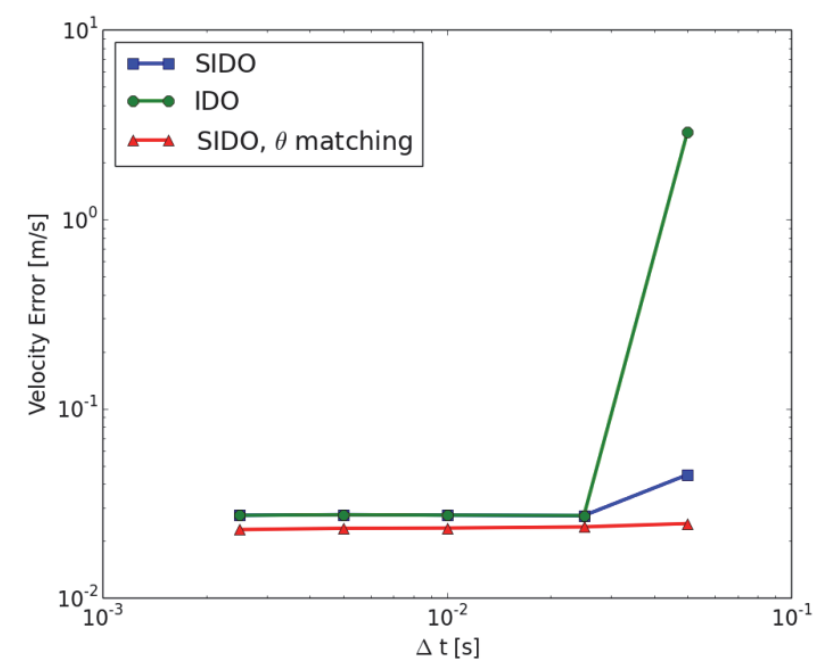

Fig. 27. Velocity convergence data for slow smooth transient in high flow regime.

This simulation is the least challenging of the cases analyzed in this paper, due to the strong stabilizing effects of friction and the weak time derivatives involved. Coupled results for SIDO with and without $\theta$ matching and for IDO are shown in Fig. 28 compared to a reference STAR-CCM+ curve. The pressure drop resulting from this transient looks quite similar to that of the fast smooth transient of the previous section, but with smaller ultimate values. The coupled solutions all match quite well with the reference CFD solution, confirming their applicability to this transient. 


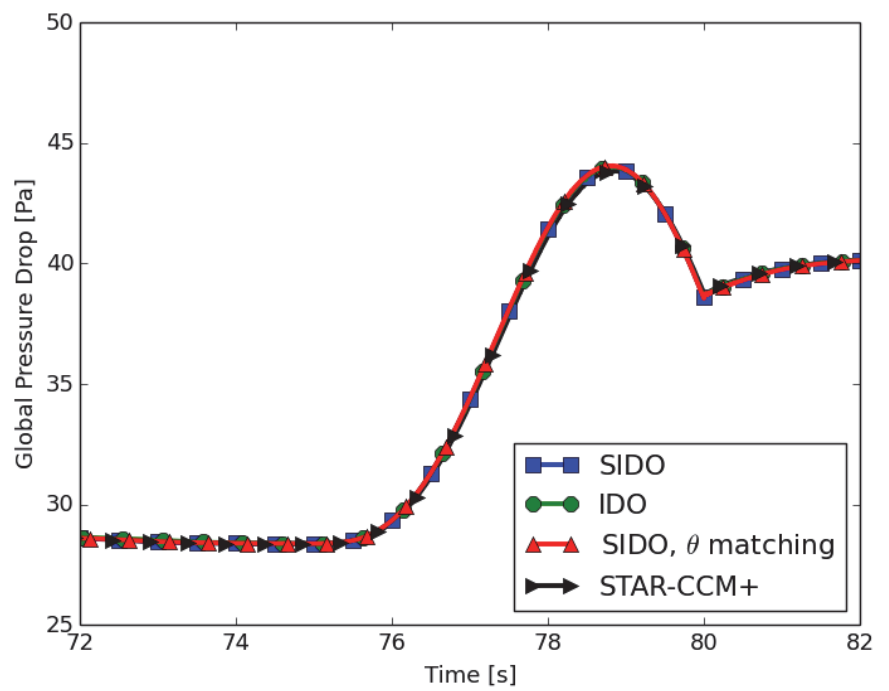

Fig. 28. Global pressure drop functions calculated with $\Delta t=. \quad s$ for the slow smooth inlet mass flow rate in the high flow regime.

\subsection{Low Flow Transient}

Low flow transients analyzed in this chapter are characterized by an initial mass flow rate of $5.0 \mathrm{~kg} / \mathrm{s}$, with Reynolds numbers for each face falling in the range $R e \in 4.48 \cdot 103,6.49 \cdot 104$. Due to the diminished stabilizing effect of frictional losses compared to the inertial pressure gradient, transients in this flow regime are significantly more challenging than their counterparts in the higher flow regime. Analogous to the previous section, three transient mass flow rates (shown in Fig. 29 (TOP), with their respective time derivatives shown in Fig. 29 (BOTTOM)) are employed to test the IDO and SIDO methods under diverse conditions. 

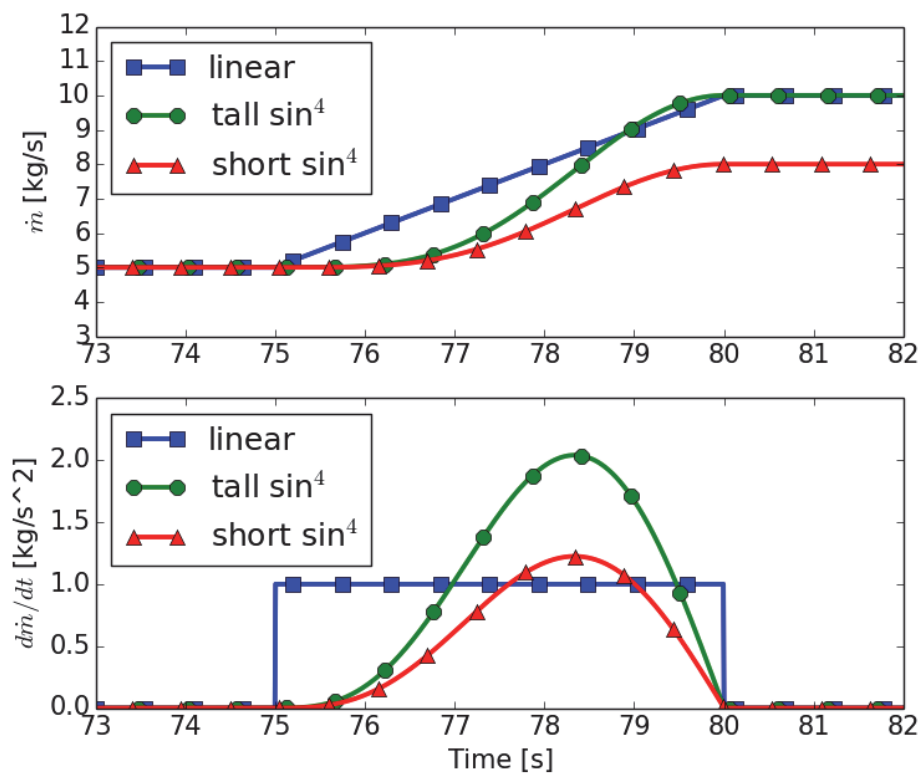

Fig. 29. (TOP) Time dependent mass flow rates used for transient test cases. (BOTTOM) derivative w.r.t. time for the two transient mass flow rates.

\subsubsection{Linear Transient}

For the linear transient in the low flow regime, Eq. 41 was applied to the flow domain inlet. Similar to $\S 4.3 .1$, this function is characterized by a discontinuous time derivative at $t=75 \mathrm{~s}$ and $t=80 \mathrm{~s}$. The magnitude of the time derivative is the same as the analogous case in the high flow regime, but the relative magnitude compared to the underlying stabilizing friction loss is much larger:

$$
\begin{gathered}
\operatorname{mint}=5.0 \mathrm{kgs}, t \leq 75.0 \mathrm{~s}, 5.0 \mathrm{kgs}+t-75.0 \mathrm{skgs} 2,75.0 \mathrm{~s}<t<80.0 \mathrm{~s}, 10.0 \mathrm{kgs}, \\
t \geq 80.0 \mathrm{~s} .
\end{gathered}
$$

Pressure convergence data are shown in Fig. 30. The test case behaves similarly to the linear transient in the high flow regime. The SIDO cases again appear to be less sensitive to the $\infty$-norm error introduced resulting from the effects described by Eq. 35. Using the $\theta$-matched VMF configuration significantly increases the $\infty$-norm error for all time steps and the 2-norm error at small time steps. 

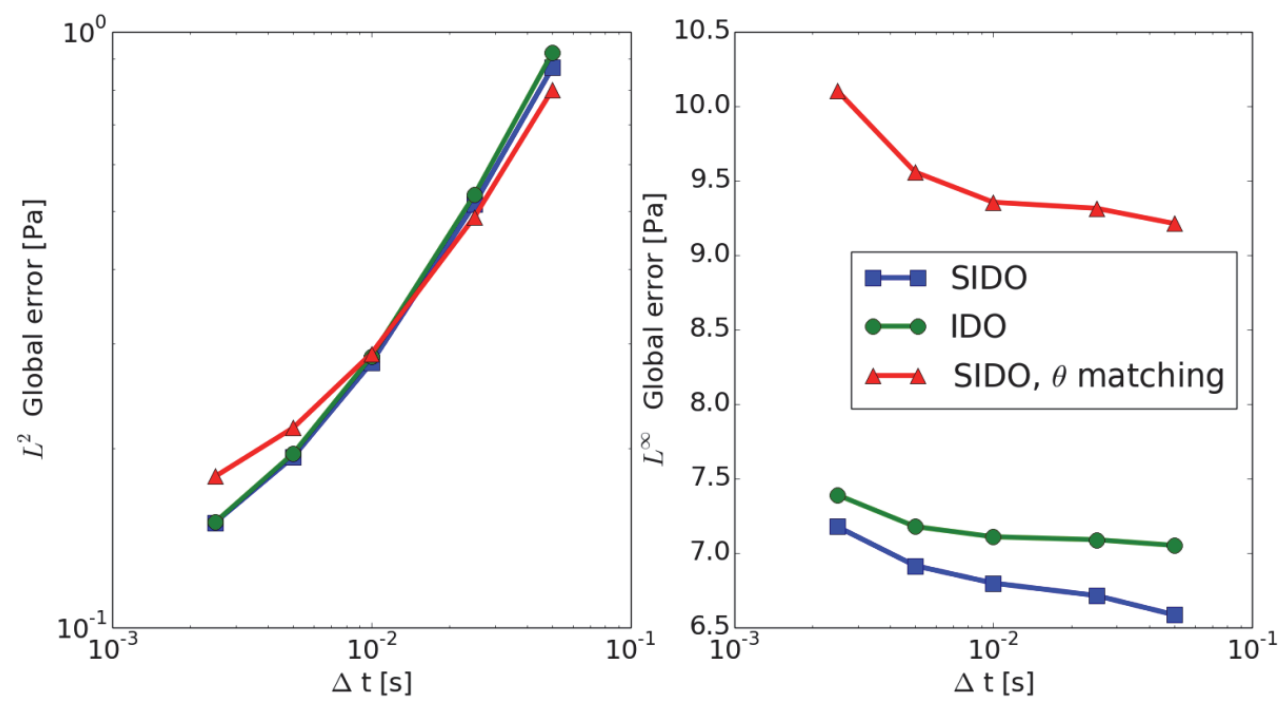

Fig. 30. Pressure convergence data for linear transient in the low flow rate regime. (LEFT) -norm error data. (RIGHT) $\infty$-norm error.

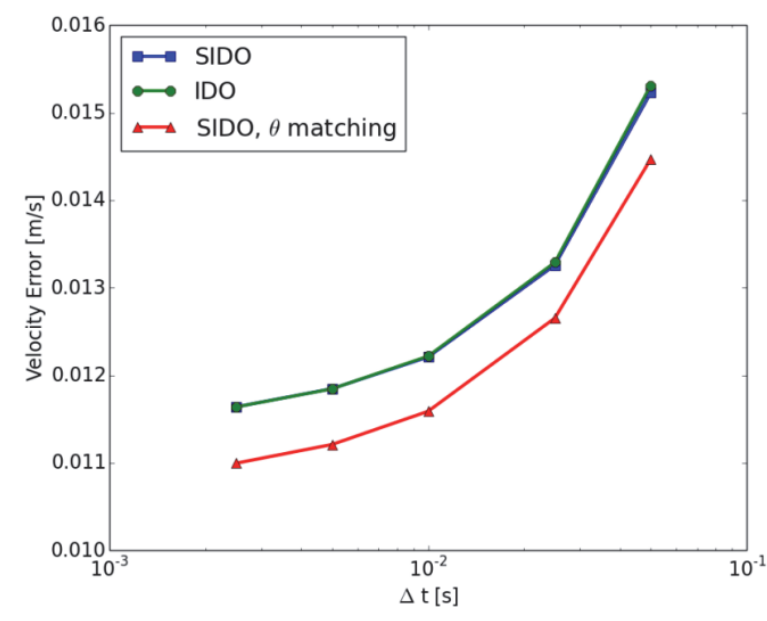

Fig. 31. Velocity convergence data for linear transient in low flow regime.

The velocity convergence data in Fig. 31 show that the $\theta$-matched VMF configuration leads to the least velocity error, as would be expected. The IDO and SIDO methods without $\theta$-matching lead to quite similar error in the velocity. Coupled results for SIDO with and without $\theta$-matching and for IDO are shown in Fig. 32, compared to a reference STAR-CCM+ curve. Similar to the linear transient in the high flow regime, the coupled results match the STAR-CCM+ results very well away from the discontinuities in the time derivative of the inlet mass flow rate at $t=75 \mathrm{~s}$ and $t=80 \mathrm{~s}$. 


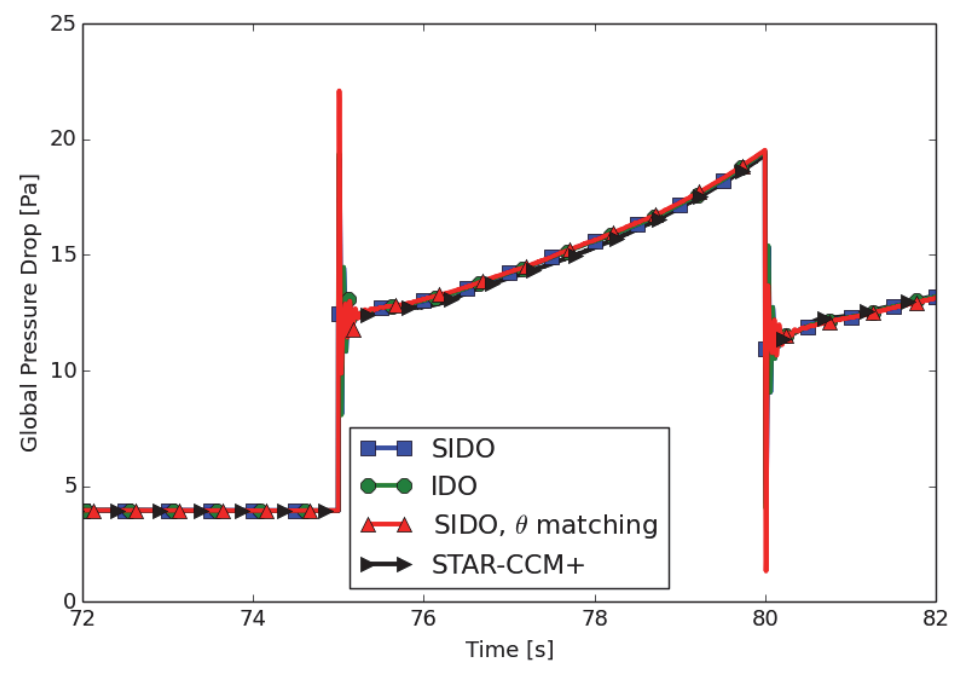

Fig. 32. Global pressure drop functions calculated with $\Delta t=. \quad s$ for the linear inlet mass flow rate in the low flow regime.

\subsubsection{Fast Smooth Transient}

For the fast smooth transient in the low flow regime, Eq. 39 was applied to the inlet of the flow domain:

$$
\begin{gathered}
\text { mint }=5.0 \mathrm{kgs}, t \leq 75.0 \mathrm{~s}, 5.0 \mathrm{kgs} 1+\sin 4 t-75.0 \mathrm{~s} 10.0 \mathrm{~s} \pi, \\
75.0 \mathrm{~s}<t<80.0 \mathrm{~s}, 10.0 \mathrm{kgs}, t \geq 80.0 \mathrm{~s} .
\end{gathered}
$$

Due to the diminished stabilizing effects of frictional loss and the high temporal derivative of mass flow rate, this transient turned out to be the most challenging test case discussed in this chapter, and indeed motivated the development of the stabilized coupling method. The data shown in Fig. 33 illustrate an unusual convergence pattern in both norms. The error for the IDO coupling implementation increases substantially at $\Delta t=0.01 \mathrm{~s}$, while the $\theta$-matched SIDO case shows a similar behavior incipient at smaller time steps. The SIDO coupling without $\theta$-matching shows much more favorable convergence behavior. 

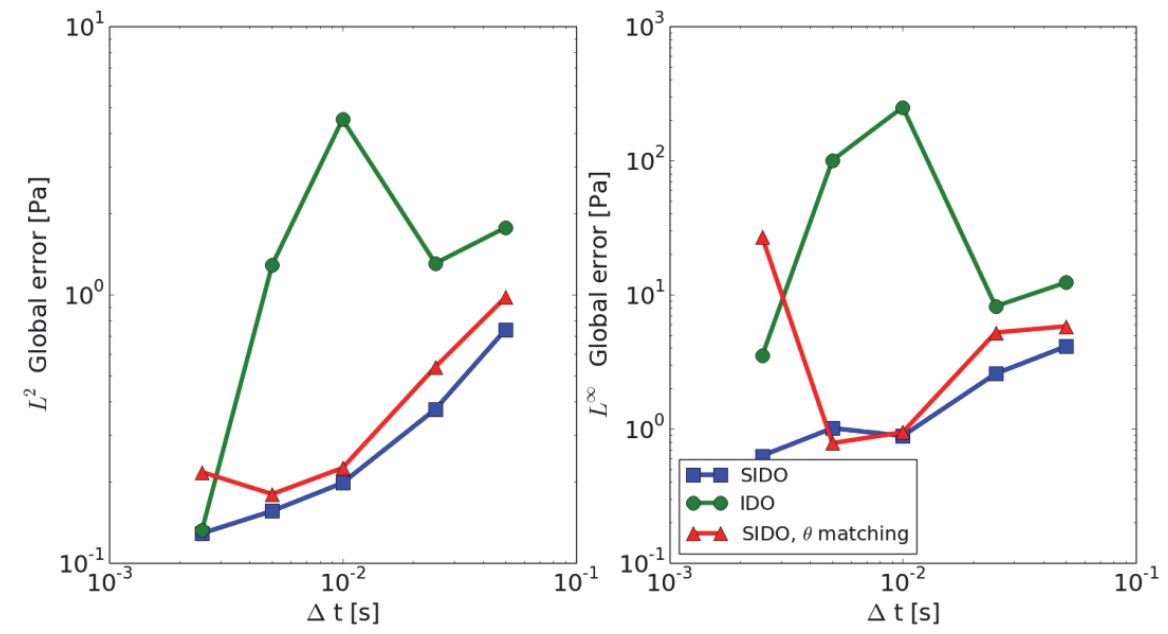

Fig. 33. Pressure convergence data for fast smooth transient in the low flow rate regime. (LEFT) norm error data. (RIGHT) $\infty$-norm error.

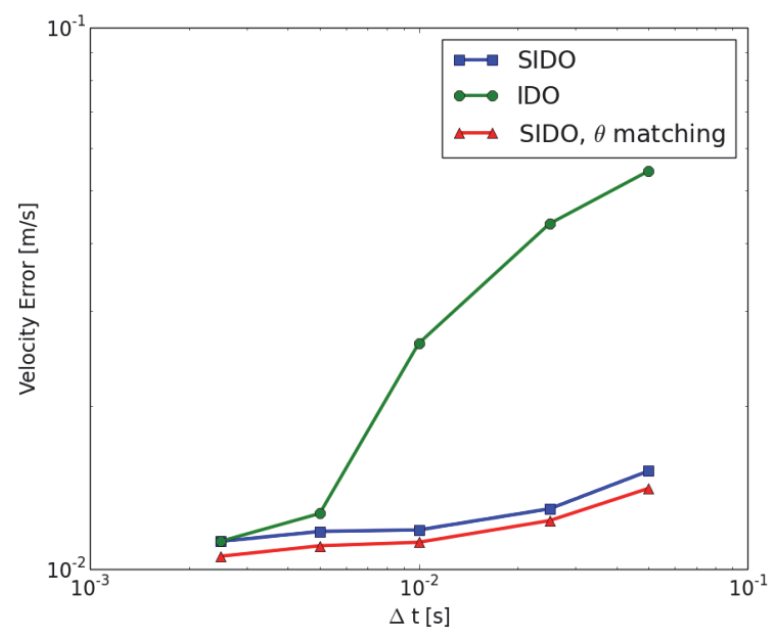

Fig. 34. Velocity convergence data for fast smooth transient in low flow regime.

The velocity error data in Fig. 34 shows a departure from the behavior observed in previous sections. Substantial error is noted for the IDO coupling case for all but the smallest of time steps. The unique behavior of the velocity data suggests that the velocity field plays an important role in the solution stability, an idea that will be corroborated by a deeper examination of the solution of the continuity equation.

The form of the convergence data is better understood upon inspection of Fig. 35, in which an instability is observed for the IDO case calculated with $\Delta t=0.01 \mathrm{~s}$. Near the end of the transient, large oscillations of high frequency appear in the pressure drop response functions that essentially destroy the usefulness of the coupling. The instability appeared to manifest at a time step of $\Delta t=0.01 \mathrm{~s}$. Close inspection of this test case revealed that certain edges were accompanied by a value of $E \Delta \Delta p$ (Eq. 22) similar in size to the total pressure drop across the edge. 


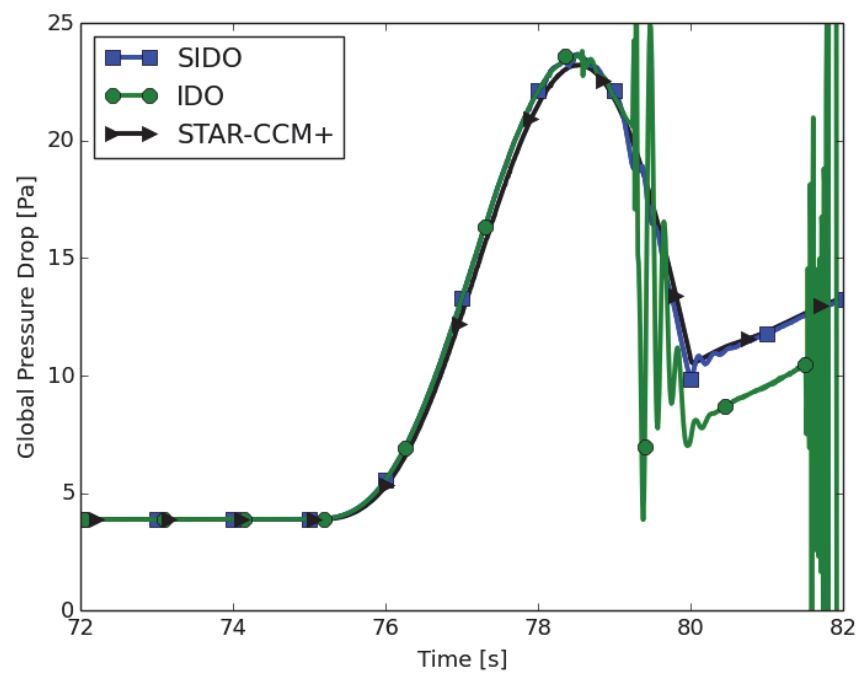

Fig. 35. Total pressure drop for the fast smooth transient in the low flow rate regime calculated with $\Delta t=$. s.

As is observed in Fig. 35, the stabilization term has almost no effect on the total pressure drop until the peak of the function around $t \approx 78.5 \mathrm{~s}$, where the SIDO and IDO curves begin to exhibit different behavior. On the downward slope, the stabilizer effect is apparent in rather dramatic fashion. A deeper discussion of continuity is beneficial to the understanding this behavior. We define a cell-based continuity function, $F$ shown in Eq. 40 :

$$
\begin{array}{cr}
F=v x-T R C A x-T R C-v x+T R C A x+T R C+v y-T R C A y-T R C-v y+T R C A y+T R C+v z-T R C A z- & 4 \\
T R C-v z+T R C A z+T R C . & 0
\end{array}
$$
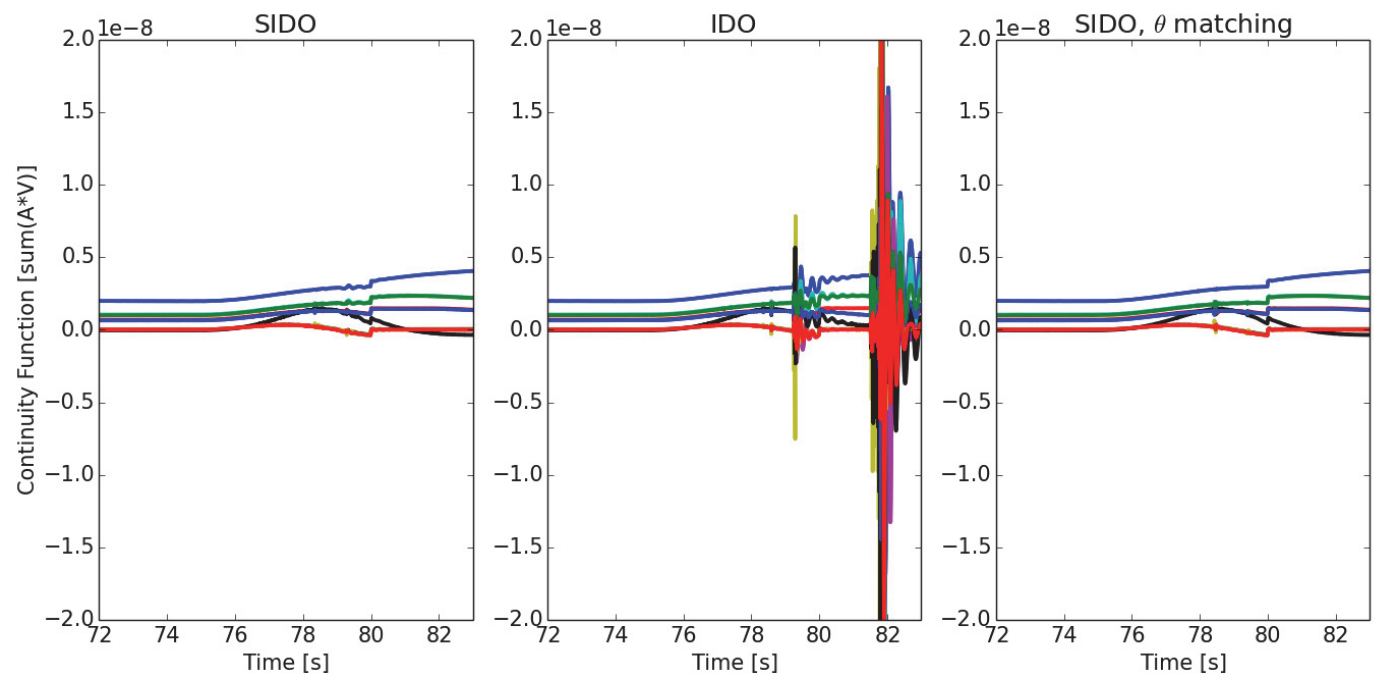

Fig. 36. Plots of continuity function $F$ for 10 (of 12 total) cells from the fast smooth transient in the low flow test case.

Data in Fig. 36 show the continuity function from Eq. 40 as calculated for 10 cells (out of 12 total) for the annular geometry. The cells with external connections (i.e. the inlet and outlet cells shown in Fig. 12) 
have been omitted for simplicity. While the $F$ values are nonzero, they are generally extremely small and within TRACE's tolerance for convergence. Upon inspection of the figure, the unstable character of the data in the center plot, calculated without stabilizer terms, can be clearly seen. As the transient progresses, the value of $F$ tends to drift, which can lead to a mass flow rate correction as TRACE attempts to enforce continuity. In the IDO case, the velocity correction is abrupt enough to cause strong perturbations to the inertial pressure drop. In the SIDO case, the stabilizer correction factors serve to balance incoming velocity and pressure data to account for the perturbation.

Coupled results for SIDO with and without $\theta$-matching and for IDO are compared in Fig. 37 to a reference standalone STAR-CCM+ solution. Even though Fig. 33 appears to show that the IDO method behaves better than SIDO with $\theta$ matching, a noticeable disruption in the coupled solution remains at the small time step. Additionally, the spike in the SIDO with $\theta$-matching convergence curve is evident in this figure as a disruption in the coupled solution near the peak of the transient.

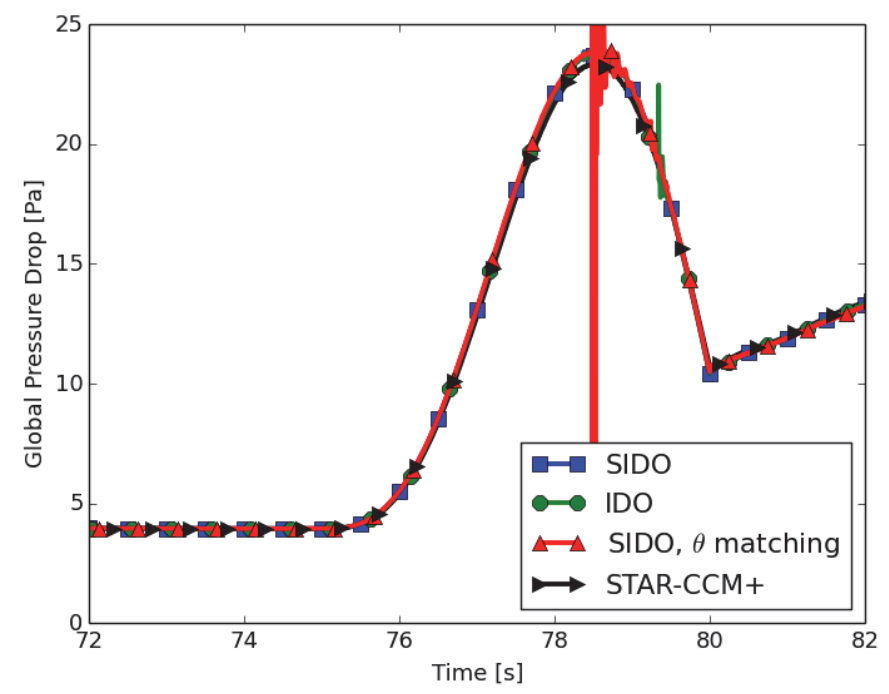

Fig. 37. Global pressure drop functions calculated with $\Delta t=. \quad s$ for the fast smooth inlet mass flow rate in the low flow regime.

\subsubsection{Slow Smooth Transient}

For the slow smooth transient in the low flow regime, Eq. 41 was applied to the inlet of the flow domain. The mass flow rate function is of the same shape as that used in the previous section, but with a smaller amplitude, leading to a smaller time derivative and a smaller ultimate flow rate. The maximum time derivative is somewhat higher than the derivative of the linear function used in $\S 4.4 .1$, but is lower on average:

$$
\begin{gathered}
\text { mint }=5.0 \mathrm{kgs}, t \leq 75.0 \mathrm{~s}, 5.0 \mathrm{kgs} 1+35 \mathrm{sin} 4 t-75.0 \mathrm{~s} 10.0 \mathrm{~s} \pi, \\
75.0 \mathrm{~s}<t<80.0 \mathrm{~s}, 8.0 \mathrm{kgs}, t \geq 80.0 \mathrm{~s} .
\end{gathered}
$$


The pressure drop convergence data in Fig. 38 and the velocity field convergence data Fig. 39 show that this test case is not plagued with the same instability as the fast smooth transient in the low flow regime, suggesting that the high time derivative of mass flow rate is a strong factor affecting the stability. Both types of error exhibit behavior similar to both the fast and slow smooth transients in the high flow regime. The relative "gentleness" of this transient does not require any abrupt velocity corrections for TRACE to maintain continuity. Additionally, there are no time derivative discontinuities to exacerbate the VMF error.
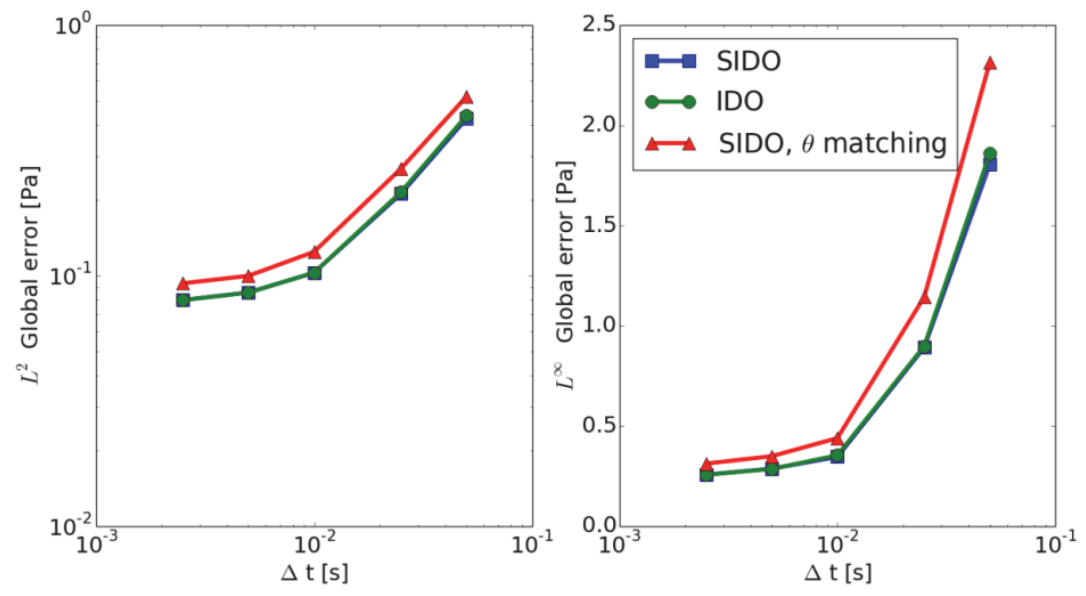

Fig. 38. Pressure convergence data for slow smooth transient in the low flow rate regime. (LEFT) norm error data. (RIGHT) $\infty$-norm error.

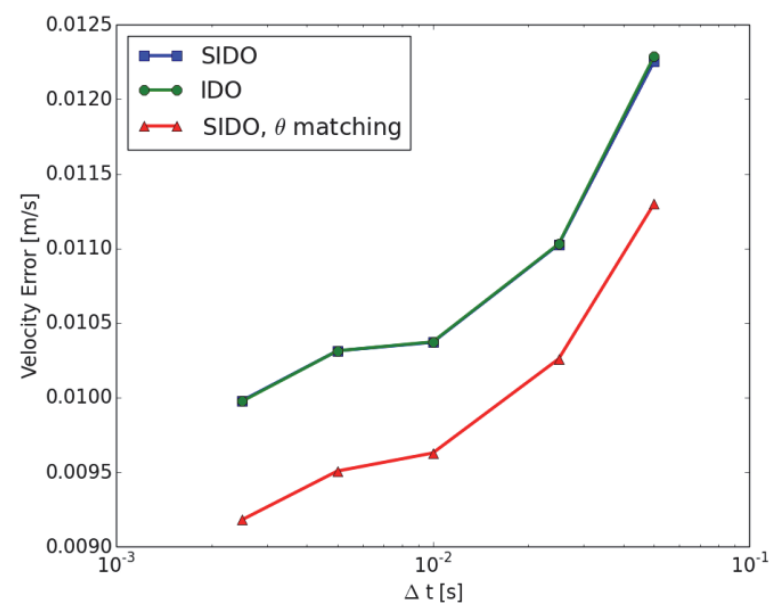

Fig. 39. Velocity convergence data for slow smooth transient in low flow regime.

Coupled results for SIDO with and without $\theta$-matching and for IDO are shown in Fig. 40 compared to a reference STAR-CCM+ curve. In stark contrast to the previous case, no disruptions are apparent in any of the coupled solutions, which match the CFD solution quite well throughout the entire transient. 


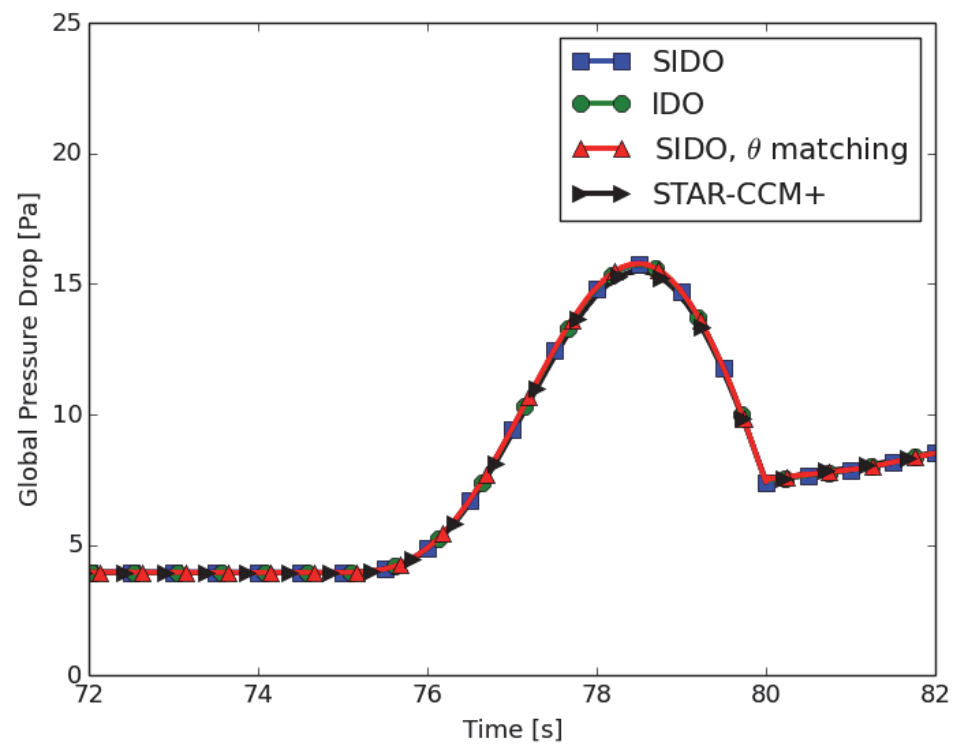

Fig. 40. Global pressure drop functions calculated with $\Delta t=. \quad s$ for the slow smooth inlet mass flow rate in the low flow regime.

\subsection{Pseudo-Implicit Transient}

A dominant portion of the error that accompanies the coupling method presented in this chapter is either caused or exacerbated by the explicit time stepping employed in the data exchange between the two coupled codes. Based on this, it is appropriate to explore the use of an implicit scheme. In general, it is not an easy task to accomplish efficient implicit coupling with closed source software such as STAR$\mathrm{CCM}+$. Performing time step backups efficiently with CFD presents an additional difficulty, especially with fine meshes. However, since the cases explored in this chapter use prescribed flow conditions, it is possible to perform a sort of "pseudo-implicit coupling" in which both the STAR-CCM+ and TRACE sides of the coupled simulations are preconfigured with identical mass flow boundary conditions (in contrast with the previous cases analyzed in this chapter, in which the flow rate functions were implemented in TRACE and passed to STAR-CCM+ through boundary coupling). This type of pseudoimplicit coupling is not possible for more realistic cases in which the coupled region is part of a broader system in which feedback mechanisms determine the flow conditions, rather than prescribed functions. However, the pseudo-implicit time stepping exercise is worthwhile to further explore the root cause of coupling error and to lay down groundwork for future efforts aimed at error reduction.

In $\S 4.3$ and $\S 4.4$, it was observed that the STAR-CCM+ solution lagged the TRACE solution by a time step. When the flow rate through the system is changing, this lag can cause considerable error through VMF interaction as estimated in Eq. 35. By performing this pseudo-implicit transient, we demonstrate that the primary error associated with the coupling method presented herein arises from the action of velocity-matching faces under explicit time-stepping schemes. Error resulting from the imperfect estimate of $\Delta P j+12 n+1-\Delta P j+12 n$ in $E \Delta \Delta p$ (Eq. 22) still remains, however. This section therefore also serves to motivate the development of advanced VMF formulations or another velocity profile coupling method altogether. 


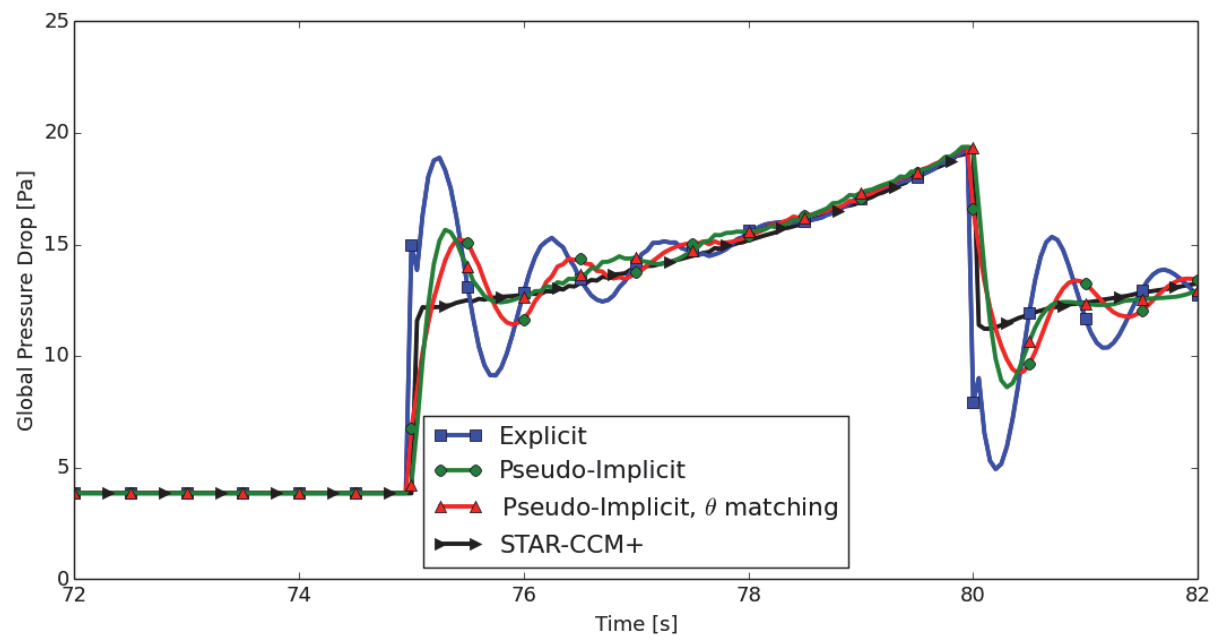

Fig. 41. Comparison of explicit and pseudo-implicit schemes for the linear transient in the low flow regime. Computed with $\Delta t=. s$.

In Fig. 41, the pseudo-implicit method is compared to the SIDO method without $\theta$-matching and explicit time stepping. The cases shown were calculated with the largest time step examined for the annulus test case $\Delta t=0.05 \mathrm{~s}$. At this time step level it is clear that the pseudo-implicit method significantly reduces the oscillations that result from discontinuities in the inertial pressure drop. Additionally, the larger oscillations predicted by Eq. 35 for $\theta$-matched cases are no longer present. Convergence data shown in Fig. 42 demonstrate that the pseudo-implicit substantially reduces error in both the 2-norm and the $\infty$ norm. As would be expected, the benefit diminishes as the time step is refined. Based on these results, it is reasonable to expect that a fully implicit method would show even further improvement.
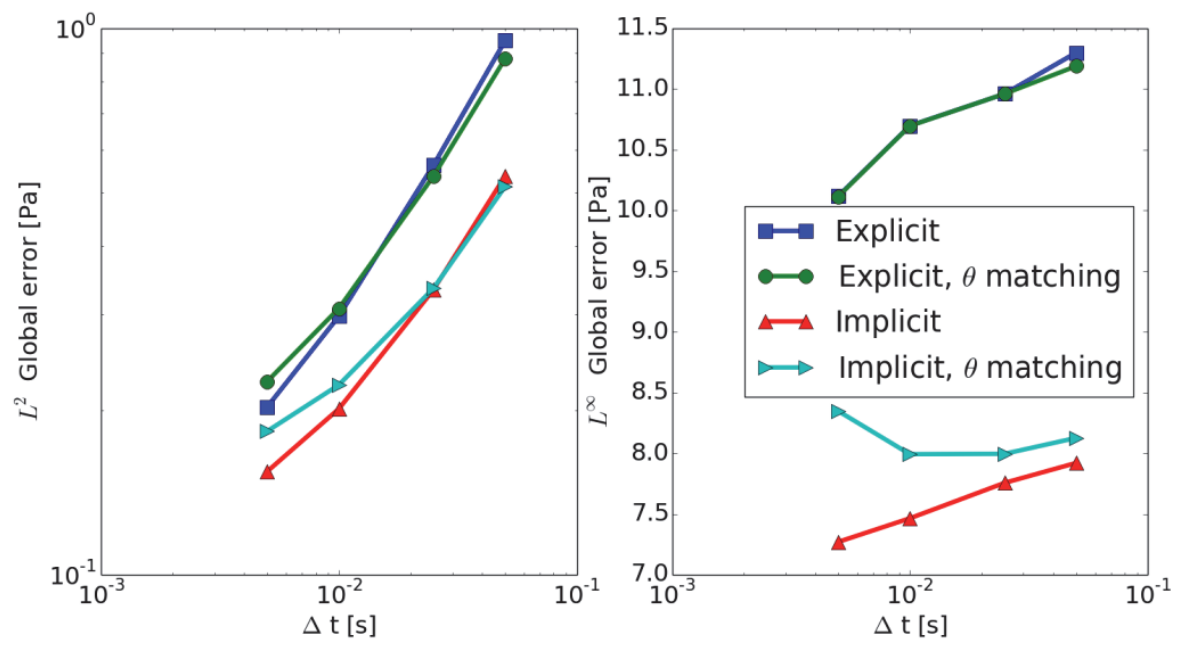

Fig. 42. Pressure drop convergence data showing effects of pseudo-implicit coupling. (LEFT) norm error data. (RIGHT) $\infty$-norm error.

\section{CONCLUSIONS}

In this paper, we described the theoretical framework of a novel domain overlapping coupling methodology between CFD and thermal-hydraulic system codes applicable to multi-dimensional regions. We showed that the STH internal solution for a 3-D component can be corrected on-the-fly to match an 
accompanying CFD solution. In order to correct the STH code's momentum equation, friction factors calculated on-the-fly from CFD data were used to account for the effects of convective acceleration, friction, and turbulence. A velocity-matching functionality was implemented to drive consistency between the STH and CFD velocity fields, thereby accounting for the effects of inertia on the momentum equation.

The application of the coupling method to a simple Cartesian flow splitter was straightforward. A relatively naïve approach to velocity-matching which ignored the coupling's effect on the nonlinear iteration of the TRACE solver, was found to be sufficient due to the simplicity of the geometry. In steady state, coupled simulations without velocity matching were found to produce CFD-like pressure drops, but STH-like velocity profiles due to the disruptive effect of the coupling on the pressure-velocity feedback intrinsic to fluid flow. Coupled simulations with velocity matching, however, produced CFD-like pressure drops and CFD-like velocity profiles, verifying the IDO method and motivating velocity-matching.

A more complex annular flow splitter test case was then simulated over a range of transients at both high and low Reynolds numbers. Transients featuring large time-derivatives of velocity in a low flow environment were identified as the most challenging cases. In these, perturbations from the velocitymatching implementation had a destabilizing influence on the coupled solution. Through analysis of the interaction between the IDO coupling method and the nonlinear iteration of the TRACE solver, correction factors were identified, leading to the SIDO method. Including azimuthal velocity-matching was found to produce more accurate velocity fields, but at the cost of reduced stability for challenging transients and slowing temporal convergence in other transients.

While this method was developed and implemented with the TRACE and STAR-CCM+ code pair, the formulation is sufficiently general to be applicable to any CFD-STH pair. The proposed coupling method stands to appreciably improve the fidelity of simulations of transients involving the simulation of multidimensional regions with complex flow phenomena, such as those present in the downcomer in the pressure vessel of a nuclear reactor.

The test systems presented in this paper were open flow systems allowing for analysis in the absence of flow feedback effects. Open loops are, however, of limited interest to industry. The feedback effects inherent to closed circuit flow systems have a strong impact on reactor safety. The SIDO method was developed bearing this in mind and application to closed loops is a subject of ongoing and future work.

\section{ACKNOWLEDGEMENTS}

This work was supported through a DOE NEUP fellowship and the US NRC grant No. NRC-HQ-12-G04-0083. 


\section{REFERENCES}

Anderson, Nolan, Yassin Hassan, and Richard Schultz. "Analysis of the hot gas flow in the outlet plenum of the very high temperature reactor using coupled RELAP5-3D system code and a CFD code." Nuclear Engineering and Design 238 (2008): 274-279.

Argonne National Laboratory. The SAS4A/SASSYS-1 Safety Analysis Code System. Edited by T. H. Fanning. ANL/NE-12/4, Nuclear Engineering Division, Argonne National Laboratory, January 31, 2012.

Aumiller, D. L., E. T. Tomlinson, and R. C. Bauer. "A Coupled RELAP5-3D/CFD Methodology with a Proof-of-Principle Calculation." Nuclear Engineering and Design 205 (2001): 83-90.

Bandini, G., et al. "Assessment of Systems Codes and their Coupling with CFD Codes in ThermalHydraulic Applications to Innovative Reactors." Nuclear Engineering and Design 281 (2015): 22-38.

Bavière, R., N. Tauveron, F. Perdu, E. Garrè, and S. Li. "A first system/CFD coupled simulation of a complete nuclear reactor transient using CATHARE2 and TRIO_U. Preliminary validation on the Phénix Reactor Natural Circulation Test." Nuclear Engineering and Design 277 (2014): 124-137.

Bergman, Theodore L., Adrienne S. Lavine, Frank P. Incropera, and David P. Dewitt. Fundamentals of Heat and Mass Transfer: Seventh Edition. John Wiley \& Sons, Inc., 2011.

Bertolotto, Davide, Annalisa Manera, Simon Frey, Horst-Michael Prasser, and Rakesh Chawla. "Singlephase mixing studies by means of a directly coupled CFD/system-code tool." Annals of Nuclear Energy 36 (2009): 310-316.

Cadinu, Francesco, and Pavel Kudinov. "Development of a "Coupling-by-Closure" Approach between CFD and System Thermal-Hydraulic Codes." Proc. The 13th International Topical Meeting on Nuclear Reactor Thermal Hydraulics (NURETH-13). Kanazawa City, Ishikawa Prefecture, Japan, 2009.

CD-Adapco. "STAR-CCM+ v8.02 Manual." 2013.

Fanning, T. H., and J. W. Thomas. Advances in Coupled Safety Modeling Using Systems Analysis and High-Fidelity Methods. ANL-GENIV-134, 2010.

Gibeling, H., and J. Mahaffy. Benchmarking Simulations with CFD to 1-D Coupling. Penn State University, Applied Research Laboratory, 2002.

Grundmann, Ulrich, and Sören Kliem. "Analyses of the OECD main steam line break benchmark with the DYN3D and ATHLET Codes." Nuclear Technology 142 (2003): 146-153.

Grunloh, Timothy P, and Annalisa Manera. "A multiscale CFD/1D-TH coupling overlapping domain methodology for thermal hydraulic systems." Transactions of the American Nuclear Society. Anaheim, CA, USA, 2014.

Grunloh, Timothy P, and Annalisa Manera. "A novel domain overlapping strategy for the multiscale coupling of CFD with 1D system codes with applications to transient flows." Annals of Nuclear Energy 90 (2016): 422-432.

Höhne, Thomas, Sören Kliem, Ulrich Rohde, and Frank-Peter Weiss. "Boron Dilution Transients During Natural Circulation Flow in PWR-Experiments anad CFD Simulations." Nuclear Engineering and Design 238 (2008): 1987-1995. 
Idaho National Engineering and Environmental Laboratory. "RELAP5-3D Code Manual Volume 1: Code Structure, System Models, and Solution Methods." Idaho Falls, Idaho, 2001.

INL. RELAP5-3D Code Manuals Revision 2.3. Idaho Falls, ID: Idaho National Lab, 2005.

Ivanov, Kostadin N., Tara M. Beam, Anthony J. Baratta, Adi Irani, and Nick Trikouros. Pressurized Water Reactor Main Steam Line Break (MSLB) Benchmark -- Volume I: Final Specifications". NEA/NSC/DOC (99) 8, U.S. Nuclear Regulatory Commission, Organization for Economic Cooperation and Development Nuclear Energy Agency, 1999.

Jeltsov, M., K. Kööp, P. Kudinov, and W. Villanueva. "Development of a Domain Overlapping Coupling Methodology for STH/CFD Analysis of Heavy Liquid Metal Thermal-Hydraulics." Proc. The 15th International Topical Meeting on Nuclear Reactor Thermal Hydraulics (NURETH-15). Pisa, Italy, 2013.

Jeong, Ji Hwan, and Byoung-Sub Han. "Coolant Flow Field in a Real Geometry of PWR Downcomer and Lower Plenum." Annals of Nuclear Energy 35 (2008): 610-619.

Joo, Han Gyu, Jae-Jun Jeong, Byung-Oh Cho, and Won Jae Lee. "Analysis of the OECD main steam line break benchmark problem using the refined cored thermal-hydraulic nodalization feature of the MARS/MASTER code." Nuclear Technology 142 (2003): 166-179.

Kozlowski, Tomasz, R. Matthew Miller, Thomas J. Downar, Douglas A. Barber, and Han Gyu Joo. "Consistent comparison of the codes RELAP5/PARCS and TRAC-M/PARCS for the OECD MSLB coupled code benchmark." Nuclear Technology 146 (2004): 15-28.

Lee, Gong Hee, Young Seok Bang, Sweng Woong Woo, and Ae Ju Cheong. "Comparative study on the effect of reactor internal structure geometry modeling methods on the prediction accuracy for PWR internal flow distribution." Annals of Nuclear Energy 70 (2014): 208-215.

Lerchl, G., H. Austregesilo, P. Schöffel, D. von der Cron, and F. Weyermann. ATHLET mod 3.0, Cycle A, User's Manual GRS-p1/Vol1, Rev.6. GRS, Germany, 2012.

Li, Wei, Xiaoli Wu, Dalin Zhang, Guanghui Su, Wenxi Tian, and Suizheng Qiu. "Preliminary study of coupling CFD code FLUENT and system code RELAP5." Annals of Nuclear Energy 73 (2014): 96-107.

Merzari, E., and H. Ninokata. "Proper orthogonal decomposition of the flow in a tight lattice rod-bundle." Nuclear Engineering and Design 241 (2011): 4621-4632.

Papukchiev, A., G. Lerchl, J. Weis, M. Scheuerer, and H. Austregesilo. "Development of coupled 1D-3D thermal-hydraulic code for nuclear power plant simulation and its application to a pressurized thermal shock scenario in PWR." Proceedings of The 14th International Topical Meeting on Nuclear Reactor Thermalhydraulics, NURETH-14. Toronto, Ontario, Canada, 2011.

Papukchiev, Angel, Georg Lerchl, Christine Waata, and Thomas Frank. "Extension of the Simulation Capabilities of the 1D System Code ATHLET by Coupling with the 3D CFD Software Package ANSYS CFX." Proc. The 13th International Topical Meeting on Nuclear Reactor Thermal Hydraulics (NURETH-13). Kanazawa City, Ishikawa Prefecture, Japan, n.d.

Papukchiev, Angel, Marti Jeltsov, Kaspar Kööp, Pavel Kudinov, and Georg Lerchl. "Comparison of different coupling CFD-STH approaches for pre-test analysis of TALL-3D experiment." Nuclear Engineering and Design 290 (2015): 135-143.

Pialla, David, et al. "Overview of the system alone and system/CFD coupled calculations of the PHENIX Natural Circulation Test within the THINS project." Nuclear Engineering and Design 290 (2015): 78-86. 
Prill, D. P., and A. G. Class. "Semi-automated proper orthogonal decomposition reduced order model non-linear analysis for future BWR stability." Annals of Nuclear Energy 67 (2014): 70-90.

Tenchine, D., et al. "Status of CATHARE code for sodium cooled fast reactors." Nuclear Engineering and Design 245 (2012): 140-152.

Theofanous, T. G., and E. A. Shabana. "Boron mixing in the lower plenum of a BWR." Nuclear Engineering and Design 126 (1991): 245-255.

U. S. Nuclear Regulatory Commission. "TRACE V5.0 Theory Manual." 2010.

Watanabe, Tadashi, Yoshinari Anoda, and Masahito Takano. "System-CFD coupled simulations of flow instability in steam generator U tubes." Annals of Nuclear Energy 70 (2014): 141-146. 